\title{
Holographic Renormalization of Two-Point Functions in Non-AdS/Non-CFT
}

\author{
Michael Haack ${ }^{1}$ and Wolfgang Mück ${ }^{2}$ \\ ${ }^{1}$ Department für Physik, Arnold Sommerfeld Zentrum für Theoretische Physik, \\ Ludwig-Maximilians-Universität, Theresienstrasse 37, 80333 München, Germany \\ ${ }^{2}$ Dipartimento di Scienze Fisiche, Università di Napoli "Federico II" and INFN, Sezione di Napoli, \\ Via Cintia, 80126 Napoli, Italy
}

Correspondence should be addressed to Michael Haack, michael.haack@physik.uni-muenchen.de

Received 19 April 2010; Accepted 14 September 2010

Academic Editor: Leopoldo P. Zayas

Copyright (C) 2010 M. Haack and W. Mück. This is an open access article distributed under the Creative Commons Attribution License, which permits unrestricted use, distribution, and reproduction in any medium, provided the original work is properly cited.

\begin{abstract}
We review recent progress on holographic renormalization in the context of the gauge-gravity correspondence when the bulk geometry is not asymptotically AdS. The prime example is the Klebanov-Strassler background, whose dual gauge theory has logarithmically running couplings at all energy scales. The presented formalism provides the counterterms necessary for obtaining finite two-point functions of the scalar operators in the corresponding dual gauge theories. The presentation is self-contained and reviews all the relevant background material concerning a gauge-invariant description of the fluctuations around holographic renormalization group backgrounds.
\end{abstract}

\section{Introduction}

Gauge/string duality offers an alternative approach to aspects of nonAbelian gauge theories that are hard to describe with conventional techniques. For example, at strong coupling, many nonAbelian gauge theories exhibit confinement, the familiar yet still somewhat mysterious phenomenon that the only finite-energy states are singlets under the color gauge group: in colliders, we never see quarks directly, only colorless hadrons. The details of confinement, and other phenomena such as chiral symmetry breaking, are difficult to capture with conventional gauge theory methods-they are inherently nonperturbative. Instead, in the dual string picture, the nonperturbative gauge theory regime is typically described by weakly coupled closed strings propagating on a space of higher dimensionality (the "bulk"), and their dynamics can be approximated by classical supergravity. In this case, one also speaks of gauge/gravity duality. 
One of the most powerful applications of gauge/gravity duality is the calculation of field theory correlation functions from the dual bulk dynamics. This idea was developed in [1-3] for superconformal gauge theories, whose gravity duals are Anti-de Sitter (AdS) spaces. Let us briefly illustrate with a simple example how such a calculation is done. The starting point is the correspondence formula [3]

$$
\mathrm{e}^{-S_{\mathrm{on}-\mathrm{sh}}[\mathfrak{s}]}=\int \Phi \Phi \mathrm{e}^{-S_{\mathrm{QFT}}[\Phi]+\int \mathcal{O}_{i} \mathfrak{s}^{i} \mathrm{~d}^{d} x}
$$

where, on the left-hand side, $S_{\text {on-sh }}[\mathfrak{s}]$ denotes the renormalized bulk on-shell action evaluated as a functional of suitably defined boundary values $\mathfrak{s}^{i}$ of the various bulk fields. The righthand side represents the QFT generating functional for connected correlation functions of the operators $\mathcal{O}_{i}$, and the $\mathfrak{s}^{i}$ play the role of sources. It is often more practical to consider the exact one-point functions of the QFT operators, which are obtained from (1.1) as

$$
\left\langle\mathcal{O}_{i}(x)\right\rangle=-\frac{\delta S_{\text {on-sh }}}{\delta \mathfrak{s}^{i}(x)}
$$
action

Let us consider a massless scalar field in $d+1=5$ dimensional AdS space, with a bulk

$$
S_{\text {bulk }}=\frac{1}{2} \int \mathrm{d}^{5} x \sqrt{g} g^{A B} \partial_{A} \phi \partial_{B} \phi
$$

where the bulk metric is taken to be

$$
\mathrm{d} s^{2}=\frac{1}{z^{2}}\left(\mathrm{~d} z^{2}+\eta_{\mu \nu} \mathrm{d} x^{\mu} \mathrm{d} x^{v}\right), \quad \mu, v=1,2,3,4 .
$$

This is AdS space with characteristic length (or "radius") $L=1$. The boundary is located at $z=0$. The equation of motion for $\phi$ is

$$
\left(\partial_{z}^{2}-\frac{3}{z} \partial_{z}+\square\right) \phi(z, \mathbf{x})=0
$$

where $\square=\partial^{\mu} \partial_{\mu}$. In momentum space, the general solution of (1.5), which is regular in the AdS bulk space (in particular, for $z \rightarrow \infty$ ), can be expressed as

$$
\phi(z, \mathbf{k})=2\left(\frac{k z}{2}\right)^{2} K_{2}(k z) \phi_{0}(\mathbf{k})
$$

where $k=\left(k^{\mu} k_{\mu}\right)^{1 / 2}$, and $K_{2}$ is a modified Bessel function. The overall constant has been chosen such that, for $z \ll 1$, one has [4]

$$
\phi(z, \mathbf{k})=\left[1-\left(\frac{k z}{2}\right)^{2}-\left(\frac{k z}{2}\right)^{4}\left(\ln \frac{k z}{2}-\psi(1)-\frac{3}{4}\right)+\cdots\right] \phi_{0}(\mathbf{k})
$$


so that $\phi_{0}$ is the boundary value, which plays the role of the source, $\mathfrak{s}$, in the dual QFT.

To calculate the on-shell action, we introduce a cutoff boundary at $z=\epsilon>0$, integrate (1.3) by parts and use the equation of motion in the bulk part, leading to

$$
S_{\text {bulk }}=-\frac{1}{2} \int \mathrm{d}^{4} x\left[z^{-4} \phi z \partial_{z} \phi\right]_{z=e}
$$

Then, substituting (1.7) and switching to momentum space yields

$$
S_{\text {bulk }}=\int \frac{\mathrm{d}^{4} k}{(2 \pi)^{4}} \phi_{0}(-\mathbf{k})\left[\left(\frac{k}{2 \epsilon}\right)^{2}+2\left(\frac{k}{2}\right)^{4}\left(\ln \frac{k \epsilon}{2}-\psi(2)\right)+\cdots\right] \phi_{0}(\mathbf{k}),
$$

where the ellipses denote terms that vanish in the near-horizon limit $\epsilon \rightarrow 0$. The two divergent terms in the near-horizon limit are removed by adding counterterms, which must take the form of local, boundary covariant functionals of the full fields $\phi$ (not of the boundary values $\left.\phi_{0}\right)$. Hence, we add

$$
S_{\text {c.t. }}=-\int \mathrm{d}^{4} x \sqrt{\gamma}\left[\frac{1}{4} \gamma^{\mu \nu} \partial_{\mu} \phi \partial_{\nu} \phi+\frac{1}{8} \ln (\kappa \epsilon)\left(\gamma^{\mu \nu} \partial_{\mu} \partial_{\nu} \phi\right)^{2}\right],
$$

where $\gamma_{\mu \nu}=\epsilon^{-2} \eta_{\mu \nu}$ is the metric on the cutoff boundary, and $\mathcal{\kappa}$ is an arbitrary constant of dimension mass. Changing $\kappa$ is, therefore, the same as adding some finite counterterms. After putting everything together and taking the near-horizon limit, we end up with the renormalized on-shell action

$$
S_{\text {on-sh }}=\frac{1}{2} \int \frac{\mathrm{d}^{4} k}{(2 \pi)^{4}} \phi_{0}(-\mathbf{k}) \mathrm{G}(\mathbf{k}) \phi_{0}(\mathbf{k}), \quad \mathbf{G}(\mathbf{k})=\frac{1}{4} k^{4} \ln \frac{k}{M}
$$

where $M$, a constant of dimension mass that depends on $\kappa$, represents the renormalization scale. In (1.11), one can recognize the generating functional for the CFT 2-point function of operators of dimension 4 .

This short example illustrates the general philosophy behind holographic renormalization (HR) and shows that the procedure has certain features in common with the usual renormalization procedure in QFT. First, the bare, regularized generating functional diverges when removing the cutoff. Second, counterterms are added at the cutoff, after which the cutoff can be removed. Third, different renormalization schemes differ from each other by finite (and local) counterterms. Last, the necessity to introduce a dimensionful constant (renormalization scale) gives rise to a scale anomaly. For a more detailed account of HR in AdS/CFT, valid in any asymptotically locally AdS (aAdS) bulk space-time and including also gravity, fermion and form fields, we refer to the extensive original literature and reviews [515]. The little "a" in aAdS is particularly important, because it allows to calculate correlators in QFTs with running couplings, where the flow away from the UV fixed point is driven either by an operator insertion or a spontaneous breaking of the scale symmetry. These are known in AdS/CFT as holographic RG flows.

There are many gauge/gravity dualities, which do not involve aAdS bulk spaces. For example, a field that has attracted much interest recently is nonrelativistic AdS/CFT, 
because of its potential applications to condensed matter physics. Let us just note that HR has been formulated for backgrounds with Schrödinger [16, 17] and Lifshitz-like scale symmetries $[18,19]$. These and other cases share the fact that their bulk backgrounds possess some anisotropic conformal infinity, which turns out to be crucial for HR [20]. Other nonconformal cases are given by $A d S_{p+2} \times S^{8-p}$ with a nonvanishing dilaton. These cases exhibit a generalized conformal structure and imply couplings that run with a power law in the UV. HR has been carried out in [21, 22] following the standard method as reviewed in [12].

In this review, we will be concerned with relativistic QFTs, whose gravity duals are formally given by so-called "fake" SUGRAs [23]. These theories describe gravity coupled to an arbitrary number of dynamical scalars, with the condition that the scalar potential can be expressed via a "superpotential". (Hence, "fake" just means that the theory is not necessarily supersymmetric, because this condition is weaker than supersymmetry. The relation between supergravity and fake supergravity was analyzed in [24, 25].) This implies the existence of BPS domain wall background solutions, which are the holographic duals of RG flows. AdS/CFT systems (with aAdS geometry) typically fall into this class of theories. What we drop, therefore, is only the requirement that the background has an asymptotic AdS region. On the QFT side of the duality, this implies to give up the existence of a UV conformal fixed point. (For this reason, we can speak of "nonAdS/nonCFT" dualities. These play a prominent role in holographic models of QCD-like theories showing features such as mesons, chiral symmetry breaking and confinement. Further details on this subject can be found in the reviews [26-28].) In contrast to the nonconformal systems mentioned earlier, in which one can perform HR based on the general asymptotic structure of the bulk space-time and fields, we will make no specific assumptions on the asymptotic geometry of the bulk. Thus, a general treatment of HR along the lines of the standard method [12] appears to be possible only on a case-by-case basis. What we would like to answer is the question whether the fake SUGRA structure of the bulk theories can be exploited to perform HR for the QFTs dual to the BPS domain wall backgrounds. The answer to this question is positive, and we review recent progress on a perturbative, or order-by-order, approach to HR. In this approach, one considers the fluctuations around the exact BPS background flow and removes the divergences order by order in the fluctuations. So far, the second-order counterterms have been constructed, which are sufficient for the calculation of two-point functions in flat backgrounds [29]. In a sense, the approach is inspired by [13, 14], where the philosophy was put forward to concentrate on the part of the counterterm action which is really necessary to calculate $n$-point functions for a given $n$, that is, the terms of $n$th order in the fluctuations. In this spirit, [29] considered the case $n=2$. It might be possible to derive the counterterms from a fully covariant expression (similar to [30]), but this has not been achieved yet. The approach is based on the gauge-invariant formalism for the dynamics of the bulk fluctuations [31-33], which we include in this review for completeness. This formalism has turned out to be very useful for the holographic calculation of correlation functions, mass spectra and scattering amplitudes, both in aAdS and nonaAdS backgrounds.

The prime example of a nonaAdS/nonCFT fake SUGRA system, which exhibits a logarithmically warped AdS geometry in the asymptotic region, is the Klebanov-Strassler (KS) solution [34], which is well approximated in the UV by the Klebanov-Tseytlin (KT) solution [35]. The fake SUGRA systems can be obtained by a consistent truncation of type-IIB SUGRA [33, 36-38] and are the gravity duals of an $S U(N+M) \times S U(N)$ gauge theory undergoing a series of Seiberg dualities, $N \rightarrow N-M$. Whereas the KT solution correctly describes the duality cascade, its bulk singularity makes it unreliable in the IR. 
The resolution of the singularity in the KS background provides a geometrical description of chiral symmetry breaking and confinement. Calculating correlation functions in these cases is much more involved, also because the procedure of HR has not been worked out yet in a systematic way similar to the aAdS case. As a result, only a few attempts to calculate correlators using the KT background have been made, compare for example, [30, 39-41], and only in [30,41] the program of HR, as reviewed in [12], was applied. A generalization of this procedure to include flavor degrees of freedom was discussed in [42]. Furthermore, calculations of mass spectra in the KS background [39, 43-47] have been done using a pragmatic approach assuming that a consistent method of HR in nonaAdS backgrounds exists.

Other systems of recent interest are derived from the Maldacena-Nunez background [48], the gravity dual of $\mathcal{N}=1$ SYM describing, in the UV, the exact NSVZ $\beta$-function [49] and a nonzero gluino condensate in the IR that breaks the chiral symmetry. In particular, socalled walking solutions have been considered [50-52], where in a certain regime the gauge coupling changes very little-it walks as opposed to runs. The walking region imitates, in many respects, a conformal fixed point, and the slightly broken scale symmetry implies the existence of a light particle. Walking Technicolor [53] is a phenomenologically interesting alternative to the Higgs sector of the Standard Model. Glueball mass spectra in backgrounds with walking couplings have been calculated in [54,55].

There is, therefore, continued interest in understanding better the various aspects of HR in nonaAdS backgrounds. As far as the calculation of correlation functions is concerned, the state of the art are the mass spectra that have been mentioned above. To put these calculations on a more solid footing and to prepare the ground for further investigations (fully momentum-dependent propagators, three-point functions, etc.), a consistent and systematic procedure of HR should be constructed.

Let us now state the main idea of the order-by-order approach. The starting point of the holographic calculation of correlation functions in AdS/CFT, which we generalize to nonaAdS configurations, is the correspondence formula (1.1). In AdS/CFT, the sources $\mathfrak{s}^{i}$ are identified with asymptotically rescaled boundary values of the on-shell bulk fields. When trying to generalize this identification, one has to be aware of some implicit assumptions that one usually uses in AdS/CFT [33]. The existence of a fixed point, where the bulk geometry would be global AdS, allows to parameterize the scalars in such a way that their origin is located at the fixed point, and that the individual scalars are the duals of boundary operators with certain scaling dimensions. The fixed point corresponds to some conformally invariant QFT (CFT). Therefore, holographic RG backgrounds that flow away from the fixed point can be considered either as the duals of deformations of the CFT by relevant operators or as the duals of vacua with spontaneously broken conformal symmetry, with finite couplings or vacuum expectation values (VEVs), respectively, which are related to the background scalars. In the nonaAdS case, such an interpretation is not directly possible. The absence of a fixed point does not allow to fix an origin or to choose some otherwise preferred parameterization of the scalar fields. On the field theory side, it is not possible to say that the RG flow is due to some operator deformation or a ground state with a VEV of some special QFT. All we seem to be able to say is that the holographic RG background is the dual of some QFT that has running couplings. (In AdS/CFT, one would simply say that the QFT under consideration is the CFT + deformations or in a spontaneously broken phase.) Just as in AdS/CFT, however, correlation functions of this QFT can be obtained by studying the dynamics of the fluctuations of the bulk fields around the holographic RG flow. For two-point functions, which we will be concerned with, it is sufficient to solve the linearized equations of motion, which have a useful form in 
the gauge-invariant formalism. The sources $\mathfrak{s}^{i}$ will be given by certain asymptotic boundary values of the on-shell bulk fluctuations.

Scheme dependence of correlation functions (but not of physical amplitudes) is a general feature of renormalization in QFT. When adding counterterms that cancel the cutoff dependent divergences, one is free to add finite counterterms, which are normally chosen such that certain renormalization conditions are satisfied at some renormalization scale. The same holds in HR in AdS/CFT. Also the order-by-order approach presented here includes scheme dependence, although in a more indirect way. The choice of scheme is reflected in certain ambiguities in the choice of the asymptotic basis functions for the fluctuations.

The rest of the review is structured as follows. In Section 2, we review the linearized bulk dynamics of a fake SUGRA system around BPS domain wall backgrounds, expressed in the language of gauge-invariant variables introduced in [31-33], and we introduce the asymptotic decomposition of the on-shell fields into dominant and subdominant basis solutions. The holographic renormalization for two-point functions following the order-byorder approach of [29] is presented in Section 3. We restrict our attention to the scalar sector, but the recipe can be extended easily to the traceless transversal fluctuations of the metric. Because the results of this part yield new insights into the pole structure of the two-point functions, we will also include the relevant updated material from [45]. Then, it will be discussed how scheme dependence is incorporated in the order-by-order approach. Section 4 is dedicated to examples with aAdS backgrounds, that serve to verify the agreement of the results of Section 3 with known results from AdS/CFT. Apart from the pure AdS case, we will consider the GPPZ [56] and the Coulomb branch (CB) flows [57, 58], which have been extensively studied in the literature. The GPPZ flow represents a massive deformation of $\mathcal{N}=4 \mathrm{SYM}$, while the CB flow is dual to a vacuum of $N=4 S Y M$, in which the conformal invariance is spontaneously broken by a VEV. This gives us enough confidence to carry on and consider the KS case in Section 5. Finally, Section 6 contains our conclusions.

\section{Bulk Dynamics}

Let us start by reviewing the equations governing the dynamics of the bulk fields $[32,33]$, which encode the information about two-point functions in holographic renormalization group flows.

The systems we consider are fake SUGRAs in $d+1$ dimensions with actions of the form

$$
S=\int \mathrm{d}^{d+1} x \sqrt{g}\left[-\frac{1}{4} R+\frac{1}{2} G_{a b} g^{M N} \partial_{M} \phi^{a} \partial_{N} \phi^{b}+V(\phi)\right]+S_{b}
$$

with $M, N=0,1, \ldots d$, and where the potential $V(\phi)$ is given in terms of a superpotential $W(\phi)$ by

$$
V(\phi)=\frac{1}{2} G^{a b} W_{a} W_{b}-\frac{d}{d-1} W^{2}
$$

We will not specify at this point the boundary terms $S_{b}$ in (2.1), as they do not affect the bulk dynamics, although they are important for holographic renormalization. We will come back to them in Section 3. In (2.1) and (2.2), $G_{a b}$ is the metric on the sigma-model target space and $G^{a b}$ is its inverse. Moreover, we used the notation $W_{a}=\partial_{a} W=\partial W(\phi) / \partial \phi^{a}$. 
Holographic renormalization group flows are described by domain wall backgrounds of the form

$$
\mathrm{d} s^{2}=\mathrm{d} r^{2}+\mathrm{e}^{2 A(r)} \eta_{\mu \nu} \mathrm{d} x^{\mu} \mathrm{d} x^{\nu}, \quad \phi^{a}=\bar{\phi}^{a}(r)
$$

with $\mu, v=1, \ldots, d$, satisfying the BPS equations

$$
\partial_{r} A=-\frac{2}{d-1} W(\bar{\phi}), \quad \partial_{r} \bar{\phi}^{a}=W^{a}(\bar{\phi}) .
$$

Linearized fluctuations around such a domain wall background are best described in a gauge invariant fashion, which we review next.

\subsection{The Sigma-Model Covariant Field Expansion}

It is our aim to study the dynamics of the fake supergravity system (2.1), (2.2) on some known backgrounds of the form (2.3), (2.4). In this section, we will expand the fields around the background, exploiting the geometric nature of the physical variables to achieve a gaugeinvariant formulation of the fluctuation dynamics.

As is well known in gravity, reparametrization invariance of space-time comes at the price of dragging along redundant metric variables together with the physical degrees of freedom. One usually attempts to reduce redundancy by gauge fixing, but such an approach causes problems for the study of fluctuations in holographic RG flows, due to the coupling between the metric and scalar fluctuations [59-61]. Thus, following [31], we will start from a clean slate keeping all metric degrees of freedom and describe in the next subsection how to isolate the physical ones.

Using the sigma-model metric $G_{a b}(\phi)$, one can define the sigma-model connection,

$$
\mathcal{G}_{b c}^{a}=\frac{1}{2} G^{a d}\left(\partial_{c} G_{d b}+\partial_{b} G_{d c}-\partial_{d} G_{b c}\right)
$$

and its curvature tensor,

$$
\mathcal{R}_{b c d}^{a}=\partial_{c} \mathcal{G}_{b d}^{a}-\partial_{d} \mathcal{G}_{b c}^{a}+\mathcal{G}_{c e}^{a} \mathcal{G}_{b d}^{\mathrm{e}}-\mathcal{G}_{d e}^{a} \mathcal{G}_{b c}^{\mathrm{e}}
$$

We also define the covariant field derivative as usual, for example,

$$
D_{a} A_{b} \equiv A_{a \mid b} \equiv \partial_{b} A_{a}-\mathcal{G}_{a b}^{c} A_{c}
$$

All indices after a bar " $\mid$ " are intended as covariant field derivatives according to (2.7). Moreover, field indices are lowered and raised with $G_{a b}$ and $G^{a b}$, respectively.

Armed with this notation, it is straightforward to expand the scalar fields in a sigmamodel covariant fashion. The naive ansatz $\phi^{a}=\bar{\phi}^{a}+\varphi^{a}$, introducing $\varphi^{a}$ simply as the coordinate difference between the points $\phi$ and $\bar{\phi}$ in field space, leads to noncovariant expressions at quadratic and higher-orders, because these $\varphi^{a}$ do not form a vector in (tangent) 


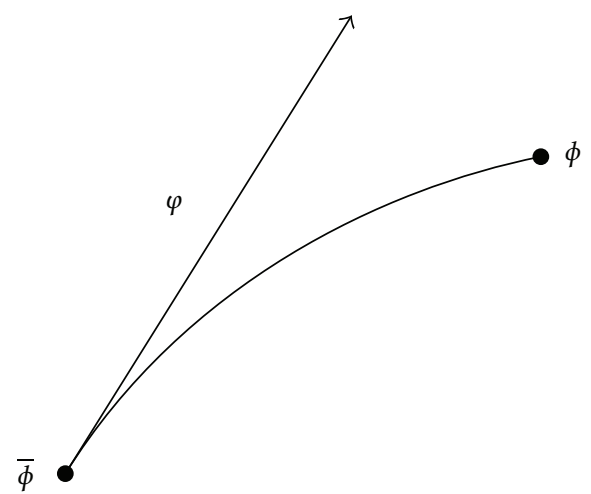

Figure 1: Illustration of the exponential map.

field space. In other words, the coordinate difference is not a geometric object. However, it is well known that a covariant expansion is provided by the exponential map, see for instance $[62,63]$,

$$
\phi^{a}=\exp _{\bar{\phi}}(\varphi)^{a} \equiv \bar{\phi}^{a}+\varphi^{a}-\frac{1}{2} \mathcal{C}_{b c}^{a} \varphi^{b} \varphi^{c}+\cdots
$$

where the higher-order terms have been omitted, and the connection $\mathcal{G}_{b c}^{a}$ is evaluated at $\bar{\phi}$. Geometrically, $\varphi$ represents the tangent vector at $\bar{\phi}$ of the geodesic curve connecting the points $\bar{\phi}$ and $\phi$, and its length is equal to the geodesic distance between $\bar{\phi}$ and $\phi$; see Figure 1 .

It is also a standard result that the components $\varphi^{a}$ coincide with the Riemann normal coordinates (RNCs) (with origin at $\bar{\phi}$ ) of the point $\phi$ (see, e.g., [63]). This fact can be used to simplify the task of writing equations in a manifestly sigma-model covariant form. Namely, given a background point $\bar{\phi}$, we can use RNCs to describe some neighborhood of it and then employ the following properties at the origin of the RNC system:

$$
\mathcal{G}_{b c}^{a}=0, \quad \mathcal{R}_{b c d}^{a}=\partial_{c} \mathcal{G}_{b d}^{a}-\partial_{d} \mathcal{G}_{b c^{\prime}}^{a}
$$

in order to express everything in terms of tensors. Because the background fields depend on $r$, we must be careful to use (2.9) only outside $r$-derivatives, but the simplifications are still significant.

Finally, let us also define a "background-covariant" derivative $D_{r}$, which acts on sigma-model tensors as, for example,

$$
D_{r} \varphi^{a}=\partial_{r} \varphi^{a}+\mathcal{C}_{b c}^{a} W^{b} \varphi^{c}
$$

If a tensor $A_{a}$ depends on $r$ only implicitly through its background dependence, then we find the identity

$$
D_{r} A_{a}(\bar{\phi})=W^{b}(\bar{\phi}) D_{b} A_{a}(\bar{\phi})
$$


The background-covariant derivative $D_{r}$ will be important in our presentation of the field equations in Section 2.4.

\subsection{Gauge Transformations and Invariants}

The form of the background solution (2.3) lends itself well to the ADM (or time-slicing) formalism for parametrizing the metric degrees of freedom, compare for example, [62, 64]. Instead of slicing in time, we will write a general bulk metric in the radially-sliced form

$$
\mathrm{d} s^{2}=\left(n^{2}+n_{\mu} n^{\mu}\right) \mathrm{d} r^{2}+2 n_{\mu} \mathrm{d} r \mathrm{~d} x^{\mu}+g_{\mu \nu} \mathrm{d} x^{\mu} \mathrm{d} x^{\nu}
$$

where $g_{\mu \nu}$ is the induced metric on the hypersurfaces of constant $r$, and $n$ and $n^{\mu}$ are the lapse function and shift vector, respectively. It will be important to us that the objects $n, n^{\mu}$ and $g_{\mu \nu}$ transform properly under coordinate transformations of the radial-slice hypersurfaces.

We can now expand the radially-sliced metric around the background configuration:

$$
\begin{gathered}
g_{\mu \nu}=\mathrm{e}^{2 A(r)}\left(\eta_{\mu \nu}+h_{\mu \nu}\right), \\
n_{\mu}=v_{\mu}, \\
n=1+v,
\end{gathered}
$$

where $h_{\mu \nu}, v_{\mu}$ and $v$ denote small fluctuations. Henceforth, we will adopt the notation that the indices of metric fluctuations, as well as of derivatives $\partial_{\mu}$, are raised and lowered using the flat (Minkowski/Euclidean) metric, $\eta_{\mu \nu}$.

Now let us turn to the question of isolating the physical degrees of freedom from the set of fluctuations $\left\{h_{v}^{\mu}, v^{\mu}, v, \varphi^{a}\right\}$ which we have introduced so far. In the earlier AdS/CFT literature one usually removed the redundancy following from diffeomorphism invariance by partial gauge fixing, that is, by placing conditions on certain components of the metric, such as $n \equiv 1, n^{\mu} \equiv 0$. And indeed, it is always possible to perform a change of coordinates which transforms the metric into a form that satisfies the gauge conditions.

Alas, as mentioned above, partial gauge fixing can create problems in coupled systems. Instead, we will obtain the equations of motion in gauge-invariant form. Let us start by considering the effect of diffeomorphisms on the fluctuation fields. We consider a diffeomorphism of the form

$$
x^{M}=\exp _{x^{\prime}}\left[\xi\left(x^{\prime}\right)\right]^{M}=x^{M}+\xi^{M}\left(x^{\prime}\right)-\frac{1}{2} \Gamma_{N P}^{M}\left(x^{\prime}\right) \xi^{N}\left(x^{\prime}\right) \xi^{P}\left(x^{\prime}\right)+\cdots,
$$

where $\xi$ is infinitesimal. Notice that we found it convenient to apply the diffeomorphism inversely, that is, we have expressed the old coordinates $x^{M}$ in terms of the new coordinates $x^{\prime M}$. The use of the exponential map implies that also the transformation laws for the fields can be written covariantly (the functions $\xi^{M}\left(x^{\prime}\right)$ are thought of as the components of a vector field). For example, a scalar field transforms as

$$
\delta \phi=\xi^{M} \partial_{M} \phi+\frac{1}{2} \xi^{M} \xi^{N} \nabla_{M} \partial_{N} \phi+\cdots,
$$


whereas a covariant tensor of rank two transforms as

$$
\begin{aligned}
\delta E_{M N}= & \xi^{L} \nabla_{L} E_{M N}+\left(\nabla_{M} \xi^{L}\right)\left(E_{L N}+\xi^{P} \nabla_{P} E_{L N}\right)+\left(\nabla_{N} \xi^{L}\right)\left(E_{M L}+\xi^{P} \nabla_{P} E_{M L}\right) \\
& +\left(\nabla_{M} \xi^{L}\right)\left(\nabla_{N} \xi^{P}\right) E_{L P}+\frac{1}{2} \xi^{P} \xi^{L}\left(\nabla_{P} \nabla_{L} E_{M N}-R_{L M P}^{S} E_{S N}-R_{L N P}^{S} E_{M S}\right) \\
& +\cdots
\end{aligned}
$$

For the metric tensor $g_{M N},(2.16)$ is simplified to

$$
\delta g_{M N}=\nabla_{M} \xi_{N}+\nabla_{N} \xi_{M}+\left(\nabla_{M} \xi^{L}\right)\left(\nabla_{N} \xi_{L}\right)-R_{M L N P} \xi^{L} \xi^{P}+\cdots
$$

Equations (2.15) and (2.16) are most easily derived using RNCs around $x^{\prime}$ and exploiting (2.9). The second-order terms in $\xi$ have been included here in order to illustrate the covariance of the transformation laws. For our purposes, the linear terms will suffice.

Splitting the fake supergravity fields into background and fluctuations, as defined in (2.13) and (2.8), the transformations (2.15) and (2.17) become gauge transformations for the fluctuations, to lowest order:

$$
\begin{gathered}
\delta \varphi^{a}=W^{a} \xi^{r}+\mathcal{O}(f), \\
\delta v=\partial_{r} \xi^{r}+\mathcal{O}(f), \\
\delta v^{\mu}=\partial^{\mu} \xi^{r}+\mathrm{e}^{2 A} \partial_{r} \xi^{\mu}+\mathcal{O}(f), \\
\delta h_{v}^{\mu}=\partial_{v} \xi^{\mu}+\partial^{\mu}\left(\eta_{v \rho} \xi^{\rho}\right)-\frac{4}{d-1} W \delta_{v}^{\mu} \xi^{r}+\mathcal{O}(f) .
\end{gathered}
$$

By $\mathcal{O}\left(f^{n}\right)$ we mean terms of order $n$ in the fluctuations $\left\{\varphi^{a}, h_{\mu \nu}, v_{\mu}, v\right\}$. Moreover, let us decompose $h_{v}^{\mu}$ as follows (in the following we will always assume $k^{2} \neq 0$ ):

$$
h_{v}^{\mu}=h_{v}^{\mathrm{TT} \mu}+\partial^{\mu} \epsilon_{v}+\partial_{v} \epsilon^{\mu}+\frac{\partial^{\mu} \partial_{v}}{\square} H+\frac{1}{d-1} \delta_{v}^{\mu} h
$$

where $h_{v}^{\mathrm{TT} \mu}$ denotes the traceless transverse part, and $\epsilon^{\mu}$ is a transverse vector. It is straightforward to obtain from (2.18)

$$
\begin{gathered}
\delta h_{v}^{\mathrm{TT} \mu}=\mathcal{O}(f), \\
\delta \epsilon^{\mu}=\Pi_{\nu}^{\mu} \xi^{v}+\mathcal{O}(f), \\
\delta H=2 \partial_{\mu} \xi^{\mu}+\mathcal{O}(f), \\
\delta h=-4 W \xi^{r}+\mathcal{O}(f) .
\end{gathered}
$$


The symbol $\Pi_{v}^{\mu}$ denotes the transverse projector,

$$
\Pi_{v}^{\mu}=\delta_{v}^{\mu}-\frac{1}{\square} \partial^{\mu} \partial_{v}
$$

The main idea of our approach is to construct gauge-invariant combinations from the fields $\left\{h_{v}^{\mathrm{TT} \mu}, \epsilon^{\mu}, h, H, v, v^{\mu}, \varphi^{a}\right\}$. Using the transformation laws (2.18) and (2.20), this is straightforward, and to lowest order, one finds the gauge-invariant fields (The choice of gauge-invariant variables is, of course, not unique, as any combination of them will be gaugeinvariant as well.)

$$
\begin{gathered}
\mathfrak{a}^{a}=\varphi^{a}+W^{a} \frac{h}{4 W}+\mathcal{O}\left(f^{2}\right), \\
\mathfrak{b}=v+\partial_{r}\left(\frac{h}{4 W}\right)+\mathcal{O}\left(f^{2}\right), \\
\mathfrak{c}=\mathrm{e}^{-2 A} \partial_{\mu} v^{\mu}+\mathrm{e}^{-2 A} \square \frac{h}{4 W}-\frac{1}{2} \partial_{r} H+\mathcal{O}\left(f^{2}\right), \\
\mathfrak{d}^{\mu}=\mathrm{e}^{-2 A} \Pi_{v}^{\mu} v^{\nu}-\partial_{r} \epsilon^{\mu}+\mathcal{O}\left(f^{2}\right), \\
\mathfrak{e}_{v}^{\mu}=h_{v}^{\mathrm{TT} \mu}+\mathcal{O}\left(f^{2}\right) .
\end{gathered}
$$

The variables $\mathfrak{c}$ and $\mathfrak{d}^{\mu}$ both arise from $\delta v^{\mu}$, which has been split into its longitudinal and transverse parts.

Although we have carried out the construction of gauge-invariant variables only to lowest order, and as we will see below, this is all that is needed, it is necessary for consistency that the preceding analysis can, in principle, be extended to higher-orders, which is indeed the case. In this context it becomes clear that the geometric nature of the field expansions, which is implied by the exponential map, is a crucial ingredient of the method.

Finally, let us prepare the ground for the arguments of the next subsection, where we will analyze the implications of gauge-invariance on the equations of motion. To be concise, we continue in a symbolic fashion. Let us consider the set of gauge-invariant fields, $I=\left\{\mathfrak{a}^{a}, \mathfrak{b}, \mathfrak{c}, \mathfrak{d}^{\mu}, \mathfrak{e}_{v}^{\mu}\right\}$. From the definitions (2.22)-(2.26) we see that there is a one-to-one correspondence between $I$ and a subset of the fluctuation fields, $Y=\left\{\varphi^{a}, v, v^{\mu}, h_{v}^{\mathrm{TT} \mu}\right\}$. We collect also the remaining fluctuation variables into a set, $X=\left\{h, H, \epsilon^{\mu}\right\}$. Henceforth, the symbols $I, X$ and $Y$ will be used also to denote the members of the corresponding sets.

One can better understand the correspondence between $I$ and $Y$ by noting that (2.22)(2.26) can be rewritten as

$$
Y=I+y(X)+\mathcal{O}\left(f^{2}\right)
$$

where $y$ is a linear functional of the fields $X$. Going to quadratic order in the fluctuations, one would find

$$
Y=I+y(X)+\alpha(X, X)+\beta(X, I)+\mathcal{O}\left(f^{3}\right),
$$


where $\alpha$ and $\beta$ are bi-linear in their arguments. Terms of the form $\gamma(I, I)$ do not appear, as they can be absorbed into $I$.

We interpret the gauge-invariant variables $I$ as the physical degrees of freedom, whereas the $(d+1)$ variables $X$ represent the redundant metric variables. This can be seen by observing that one can solve the transformation laws (2.20) for the generators $\xi^{M}$, which yields equations of the form

$$
\xi^{M}=z^{M}(\delta X)+\mathcal{O}\left(f^{2}\right)=\delta z^{M}(X)+\mathcal{O}\left(f^{2}\right),
$$

with $z^{M}$ being a linear functional.

\subsection{Einstein's Equations and Gauge Invariance}

It is our aim to cast the equations of motion into an explicitly gauge-invariant form. This means that the final equations should contain only the variables $I$ and make no reference to $X$ and $Y$. Reparametrization invariance suggests that this should be possible, and we will establish the precise details in this subsection.

Let us consider Einstein's equations, symbolically written as

$$
E_{M N}=0,
$$

but it is clear that the arguments given below hold also for the equations of motion for the scalar fields. To start, let us expand the left-hand side of (2.30) around the background solution, which yields, symbolically,

$$
E_{M N}=E_{M N}^{(1) 1}(X)+E_{M N}^{(1) 2}(Y)+E_{M N}^{(2) 1}(X, X)+E_{M N}^{(2) 2}(X, Y)+E_{M N}^{(2) 3}(Y, Y)+\mathcal{O}\left(f^{3}\right) .
$$

Here, $E^{(1)}$ and $E^{(2)}$ denote linear and bilinear terms, respectively. The background equations are satisfied identically. Substituting $I$ for $Y$ using (2.28) yields

$$
E_{M N}=\widetilde{E}_{M N}^{(1) 1}(X)+E_{M N}^{(1) 2}(I)+\widetilde{E}_{M N}^{(2) 1}(X, X)+\widetilde{E}_{M N}^{(2) 2}(X, I)+E_{M N}^{(2) 3}(I, I)+\mathcal{O}\left(f^{3}\right) .
$$

Notice that the functionals $E^{(1) 2}$ and $E^{(2) 3}$ are unchanged ( $Y$ is just replaced by $I$ ), whereas the others are modified by the $X$-dependent terms of (2.28), which we indicate by adorning them with a tilde. For example, $\widetilde{E}^{(2) 2}$ receives contributions from $E^{(2) 2}, E^{(2) 3}$ and $E^{(1) 2}$.

In order to simplify (2.32), we consider its transformation under the diffeomorphism (2.14). On the one hand, from the general transformation law of tensors (2.16) we find, using also (2.29), that it should transform as

$$
\delta E_{M N}=\left[\partial_{M} \delta z^{L}(X)\right] E_{L N}+\left[\partial_{N} \delta z^{L}(X)\right] E_{M L}+\delta z^{L}(X) \partial_{L} E_{M N}+\mathcal{O}\left(f^{3}\right) .
$$


On the other hand, the variation of (2.32) is

$$
\delta E_{M N}=\widetilde{E}_{M N}^{(1) 1}(\delta X)+2 \widetilde{E}_{M N}^{(2) 1}(\delta X, X)+\widetilde{E}_{M N}^{(2) 2}(\delta X, I)+\mathcal{O}\left(f^{3}\right) .
$$

Let us compare (2.33) and (2.34) order by order. The absence of first-order terms on the righthand side of (2.33) implies that

$$
\widetilde{E}_{M N}^{(1) 1}(X)=0 .
$$

It can easily be checked that this is indeed the case. Then, substituting $E_{M N}=E_{M N}^{(1) 2}(I)+\mathcal{O}\left(f^{2}\right)$ into the right-hand side of (2.33) yields

$$
\delta E_{M N}=\delta\left\{\left[\partial_{M} z^{L}(X)\right] E_{L N}^{(1) 2}(I)+\left[\partial_{N} z^{L}(X)\right] E_{M L}^{(1) 2}(I)+z^{L}(X) \partial_{L} E_{M N}^{(1) 2}(I)\right\}+\mathcal{O}\left(f^{3}\right) .
$$

Comparing (2.36) with the second-order terms of (2.34), we obtain

$$
\begin{gathered}
\widetilde{E}_{M N}^{(2) 1}(X, X)=0, \\
\widetilde{E}_{M N}^{(2) 2}(X, I)=\left[\partial_{M} z^{L}(X)\right] E_{L N}^{(1) 2}(I)+\left[\partial_{N} z^{L}(X)\right] E_{M L}^{(1) 2}(I)+z^{L}(X) \partial_{L} E_{M N}^{(1) 2}(I) .
\end{gathered}
$$

Hence, we find that a simple expansion of Einstein's equations yields gauge-dependent second-order terms, but they contain the (gauge-independent) first order equation, and so can consistently be dropped. Happily, we arrive at the following equation, which involves only $I$ :

$$
E_{M N}^{(1) 2}(I)+E_{M N}^{(2) 3}(I, I)+\mathcal{O}\left(f^{3}\right)=0 .
$$

The argument generalizes recursively to higher-orders. One will find that the gaugedependent terms of any given order can be consistently dropped, because they contain the equation of motion of lower orders.

Equation (2.38) and its higher-order generalizations are obtained using the following recipe:

Expand the equations of motion to the desired order dropping the fields $X$ and replacing every field $Y$ by its gauge-invariant counterpart $I$.

This rule is summarized by the following substitutions:

$$
\varphi^{a} \longrightarrow \mathfrak{a}^{a}, \quad v \longrightarrow \mathfrak{b}, \quad \mathrm{e}^{-2 A} \mathcal{v}^{\mu} \longrightarrow \mathfrak{d}^{\mu}+\frac{\partial^{\mu}}{\square} \mathfrak{c}, \quad h_{v}^{\mu} \longrightarrow \mathfrak{e}_{v}^{\mu}
$$

Since $\mathfrak{e}_{v}^{\mu}$ is traceless and transverse, the calculational simplifications arising from (2.39) are considerable.

Let us conclude with the remark that, although the rules (2.39) can be interpreted as the gauge choice $X=0$, the equations we found are truly gauge invariant. 


\subsection{Equations of Motion}

In this section, we will put into practice what we have just learned. The equations of motion that follow from the action (2.1) are

$$
\nabla^{2} \phi^{a}+\mathcal{G}_{b c}^{a} g^{M N}\left(\partial_{M} \phi^{b}\right)\left(\partial_{N} \phi^{c}\right)-V^{a}=0
$$

for the scalar fields, and Einstein's equations

$$
E_{M N}=-R_{M N}+2 G_{a b}\left(\partial_{M} \phi^{a}\right)\left(\partial_{N} \phi^{b}\right)+\frac{4}{d-1} g_{M N} V=0
$$

Notice that we use the opposite sign convention for the curvature with respect to [31, 32].

We are interested in the physical, gauge-invariant content of (2.40) and (2.41) to quadratic order in the fluctuations around an RG flow background of the form (2.3), (2.4). As we have learned in the last section, it is obtained by expanding the fields according to (2.13) and (2.8) and then applying the substitution rules (2.39). Since we defined the expansion (2.8) geometrically, it is assured that we will obtain sigma-model covariant expressions. To carry out this calculation in practice, it is easiest to use RNCs at a given point in field space, so that one can use the relations (2.9) outside $r$-derivatives.

In the following, we will present the linearized equations of motion. We just collect the results without going into details of the derivation. For intermediate steps we refer the reader to the appendices $B$ and $C$ of [33]. (Note that we use a different notation for the indices here than in [33]. The $d+1$ dimensional indices are denoted by $M, N$ here and by $\mu, v$ there, whereas the $d$ dimensional indices are denoted by $\mu, v$ here and by $i, j$ there.) Let us start with the equation of motion for the scalar fields (2.40), which gives rise to the following fluctuation equation:

$$
\left[D_{r}^{2}-\frac{2 d}{d-1} W D_{r}+\mathrm{e}^{-2 A} \square\right] \mathfrak{a}^{a}-\left(V_{\mid c}^{a}-\mathcal{R}_{b c d}^{a} W^{b} W^{d}\right) \mathfrak{a}^{c}-W^{a}\left(\mathfrak{c}+\partial_{r} \mathfrak{b}\right)-2 V^{a} \mathfrak{b}=0 .
$$

Second, the normal component of Einstein's equations gives rise to

$$
-4 W \mathfrak{c}+4 W_{a}\left(D_{r} \mathfrak{a}^{a}\right)-4 V_{a} \mathfrak{a}^{a}-8 V \mathfrak{b}=0 .
$$

Third, the mixed components of (2.41) yield

$$
-\frac{1}{2} \square \mathfrak{d}_{\mu}-2 W \partial_{\mu} \mathfrak{b}-2 W_{a} \partial_{\mu} \mathfrak{a}^{a}=0
$$

The appearance of the fields $\mathfrak{a}^{a}, \mathfrak{b}, \mathfrak{c}$ and $\mathfrak{d}^{\mu}$ on the left-hand sides of (2.42)-(2.44) seems to indicate the coupling between the fluctuations of active scalars (nonzero $W_{a}$ ) to those of the metric, which is familiar from the AdS/CFT calculation of two-point functions in the literature. However, the gauge-invariant formalism resolves this issue, because (2.43) and (2.44) can be solved algebraically (in momentum space) for the metric fluctuations $\mathfrak{b}, \mathfrak{c}$ 
and $\mathfrak{d}^{\mu}$, so that the coupling of metric and scalar fluctuations at linear order is completely disentangled. One easily obtains (using our assumption $k^{2} \neq 0$ )

$$
\begin{gathered}
\mathfrak{b}=-\frac{1}{W} W_{a} \mathfrak{a}^{a}, \\
\mathfrak{c}=\frac{W_{a}}{W}\left(\delta_{b}^{a} D_{r}-W_{\mid b}^{a}+\frac{W^{a} W_{b}}{W}\right) \mathfrak{a}^{b}, \\
\mathfrak{d}_{\mu}=0 .
\end{gathered}
$$

We proceed by substituting (2.45) and (2.46) into (2.42), using also the identities

$$
\begin{gathered}
V^{a}=W^{a \mid b} W_{b}-\frac{2 d}{d-1} W W^{a}, \\
V_{\mid c}^{a}=D_{r} W_{\mid c}^{a}+R_{b c d}^{a} W^{b} W^{d}+W^{a \mid b} W_{b \mid c}-\frac{2 d}{d-1}\left(W^{a} W_{c}+W W_{\mid c}^{a}\right),
\end{gathered}
$$

which follow from (2.2) and (2.4), and we end up with the second-order differential equation

$$
\left[\left(\delta_{b}^{a} D_{r}+M_{b}^{a}-\frac{2 d}{d-1} W \delta_{b}^{a}\right)\left(\delta_{c}^{b} D_{r}-M_{c}^{b}\right)+\delta_{c}^{a} \mathrm{e}^{-2 A} \square\right] \mathfrak{a}^{c}=0
$$

where we introduced the matrix

$$
M_{b}^{a}=W_{\mid b}^{a}-\frac{W^{a} W_{b}}{W} .
$$

Equation (2.49) is the main result of the gauge-invariant approach and governs the dynamics of scalar fluctuations around generic Poincaré-sliced domain wall backgrounds.

Let us also consider the tangential components of (2.41). Because of Bianchi's identity, their trace and divergence are implied by (2.42), (2.43), and (2.44), which is easily checked at linear order. Thus, we can use the traceless transverse projector,

$$
\Pi_{v \lambda}^{\mu \kappa}=\frac{1}{2}\left(\Pi^{\mu \kappa} \Pi_{v \lambda}+\Pi_{\lambda}^{\mu} \Pi_{v}^{\kappa}\right)-\frac{1}{d-1} \Pi_{v}^{\mu} \Pi_{\lambda^{\prime}}^{\kappa}
$$

in order to obtain the independent components. This yields

$$
\left(\partial_{r}^{2}-\frac{2 d}{d-1} W \partial_{r}+\mathrm{e}^{-2 A} \square\right) \mathfrak{e}_{v}^{\mu}=0
$$

As expected, the physical fluctuations of the metric satisfy the equation of motion of a massless scalar field.

In the following, we focus on the scalar field equation (2.49). Let $n_{s}$ be the number of scalar fields (components of $\mathfrak{a}$ ). As in [45], we will assume the existence of a set of $2 n_{s}$ independent solutions of (2.49), which are defined as power series in $k^{2}$ (in momentum 
space), with $r$-dependent coefficients that are more and more suppressed with increasing powers of $k^{2}$. (This is tantamount to demanding that the warp function $A(r)$ grows without limit for $r \rightarrow \infty$, so that $\mathrm{e}^{-2 A} k^{2}$ in (2.49) can be regarded as a correction in the asymptotic region.) Moreover, the leading term (for large $r$ ) in each solution should be independent of $k^{2}$. In position space, $k^{2}$ simply translates to the operator $-\square$. Amongst these solutions, one can distinguish between $n_{s}$ asymptotically dominant solutions $\widehat{\mathfrak{a}}_{i}\left(i=1, \ldots, n_{s}\right)$ and $n_{s}$ subdominant solutions $\breve{a}_{i}$ with respect to their behaviour at large $r$. Including the field index, we will interpret $\widehat{\mathfrak{a}}_{i}^{a}$ and $\check{\mathfrak{a}}_{i}^{a}$ as $n_{s} \times n_{s}$ matrices. A regularity condition in the bulk interior, that is,

$$
\text { Regularity condition : }\left(\mathfrak{a}^{a} G_{a b} \mathfrak{a}^{b}\right)_{r=I R}<\infty \text {, }
$$

allows only for $n_{s}$ independent regular combinations of the asymptotic basis solutions. Hence, we will decompose a general regular solution of (2.49) into

$$
\mathfrak{a}^{a}(r, x)=\widehat{\mathfrak{a}}_{i}^{a}\left(r,-\square_{x}\right) \mathfrak{s}^{i}(x)+\check{\mathfrak{a}}_{i}^{a}\left(r,-\square_{x}\right) \mathfrak{r}^{i}(x),
$$

where $\mathfrak{s}^{i}$ and $\mathfrak{r}^{i}$ are called the source and response coefficients, respectively, and $\square_{x}=$ $\eta^{\mu \nu}\left(\partial / \partial x^{\mu}\right)\left(\partial / \partial x^{\nu}\right)$. The bulk regularity condition uniquely determines the (functional) dependence of the responses $\mathfrak{r}^{i}$ on the sources $\mathfrak{s}^{i}$ and gives rise to the nonlocal information for the two-point functions of the dual operators.

Throughout the paper, we will consider mostly the analogue of (2.54) in momentum space, sometimes omitting the dependence on $k$. Moreover, a $\cdot$ will be used to denote the inner product in field space, or the contraction of field space indices, for example, $\mathfrak{a} \cdot \mathfrak{b}=\mathfrak{a}^{a} G_{a b} \mathfrak{b}^{b}$.

\section{Perturbative Holographic Renormalization}

\subsection{Scalar Two-Point Functions}

In this section, we will present the general formalism for obtaining finite, renormalized twopoint functions for the QFT operators that are dual to the bulk scalar fields. Our starting point is the following action, which is quadratic in the fluctuations and encodes the bulk field equations (2.49),

$$
S=\frac{1}{2} \int \mathrm{d}^{d+1} x \mathrm{e}^{d A}\left\{\left[\left(D_{r}-M\right) \mathfrak{a}\right] \cdot\left[\left(D_{r}-M\right) \mathfrak{a}\right]+\mathrm{e}^{-2 A} \partial_{\mu} \mathfrak{a} \cdot \partial^{\mu} \mathfrak{a}\right\}+\frac{1}{2} \int \mathrm{d}^{d} x \mathrm{e}^{d A} \mathfrak{a} \cdot U \cdot \mathfrak{a},
$$

with some symmetric counterterm matrix $U$, which is a local operator that will be specified in a moment. The bulk integral in (3.1) is to be understood with a cutoff $r_{0}$, where also the boundary counterterm is evaluated. It follows that the variation of the on-shell action with respect to a variation of the boundary value $\mathfrak{a}^{a}\left(r_{0}\right)$ is given by

$$
\frac{\delta S_{\text {on-sh }}}{\delta \mathfrak{a}^{a}}=\mathrm{e}^{d A}\left(D_{r}-M+U\right) \mathfrak{a}_{a}
$$


where the right-hand side is evaluated at $r=r_{0}$. Let us define the counterterm matrix as

$$
U_{a b}=M_{a b}-\frac{1}{2}\left[\left(D_{r} \widehat{\mathfrak{a}}\right)_{a i}\left(\widehat{\mathfrak{a}}^{-1}\right)_{b}^{i}+\left(D_{r} \widehat{\mathfrak{a}}\right)_{b i}\left(\widehat{\mathfrak{a}}^{-1}\right)_{a}^{i}\right]
$$

where $\left(\widehat{\mathfrak{a}}^{-1}\right)_{a}^{i}$ is the inverse of the matrix $\widehat{\mathfrak{a}}_{i}^{a}$, defined in momentum space as a series in $k^{2}$, or equivalently, in position space as a series in $-\square$. We will see momentarily that this definition leads to finite one- and two-point functions. We also note the following subtlety. The counterterm in (3.1) needs to be local in the fields, which means that $U_{a b}$ should be a polynomial in $k^{2}$ (in momentum space) or $-\square$ (in position space). The assumptions made in Section 2 imply that $U_{a b}$ is a series in $k^{2}$. However, we also assumed that the coefficients of the series $\widehat{a}$ with increasing powers of $k^{2}$ are suppressed for large $r$ due to the factor $\mathrm{e}^{-2 A(r)}$, so that we can truncate the series in (3.3) to some polynomial, because the terms thus neglected vanish in the large- $r$ limit. Hence, strictly speaking, the counterterm operator $U_{a b}$ in (3.1) is a polynomial truncation of (3.3).

Before deriving the two-point function, let us also introduce the following matrices:

$$
\begin{aligned}
& \tilde{Z}_{i j}=\mathrm{e}^{d A}\left[\left(D_{r} \widehat{\mathfrak{a}}\right)_{i} \cdot \widehat{\mathfrak{a}}_{j}-\widehat{\mathfrak{a}}_{i} \cdot\left(D_{r} \widehat{\mathfrak{a}}\right)_{j}\right], \\
& Z_{i j}=\mathrm{e}^{d A}\left[\left(D_{r} \widehat{\mathfrak{a}}\right)_{i} \cdot \breve{\mathfrak{a}}_{j}-\widehat{\mathfrak{a}}_{i} \cdot\left(D_{r} \breve{\mathfrak{a}}\right)_{j}\right], \\
& z_{i j}=\mathrm{e}^{d A}\left[\left(D_{r} \breve{\mathfrak{a}}\right)_{i} \cdot \breve{\mathfrak{a}}_{j}-\breve{\mathfrak{a}}_{i} \cdot\left(D_{r} \breve{\mathfrak{a}}\right)_{j}\right] .
\end{aligned}
$$

These matrices are independent of $r$, as one can show from the field equation (2.49). This implies that $z_{i j}$ should be identically zero, as the subdominant solutions vanish fast enough asymptotically. (This is not necessarily the case if there are two or more bulk scalars with mass $m^{2}=2(2-d)$, which, in the aAdS-case, would be dual to operators of dimension $\Delta=2$. If at least two of these scalar fields are present and the background is not aAdS, one has to check more carefully whether $z_{i j}$ indeed vanishes. We will assume this in the following, as it simplifies our final result. In all the examples we are considering later, this issue does not play any role.) Furthermore, they are functions of $k^{2}$ or $-\square$, depending on whether one works in momentum or position space.

Combining (3.2) with the decomposition (2.54), the (linear term of) the exact onepoint function (1.2), in momentum space, takes the form (The subscript 1 on the left-hand side indicates that these are just the terms linear in the fluctuations.)

$$
\left\langle\mathcal{O}_{i}(k)\right\rangle_{1}=-\lim _{r \rightarrow \infty} \mathrm{e}^{d A(r)}\left[\widehat{\mathfrak{a}}_{i}+\check{\mathfrak{a}}_{j} \frac{\partial \mathfrak{r}^{j}}{\partial \mathfrak{s}^{i}}(k)\right] \cdot\left(D_{r}-M+U\right)\left[\widehat{\mathfrak{a}}_{l} \mathfrak{s}^{l}(k)+\check{\mathfrak{a}}_{l} \mathfrak{l}^{l}(k)\right],
$$

where, for the sake of brevity, we have omitted the dependence of the asymptotic solutions $\widehat{\mathfrak{a}}$ and $\breve{a}$ on $r$ and $k^{2}$. 
Substituting (3.3) into (3.5) and using the matrices (3.4), after some algebra one obtains

$$
\begin{aligned}
\left\langle\mathcal{O}_{i}(k)\right\rangle_{1}= & Z_{i j} \mathfrak{r}^{j}+\frac{1}{2} \tilde{Z}_{i j \mathfrak{s}^{j}}+\frac{1}{2} z_{j k} \frac{\partial \mathfrak{r}^{j}}{\partial \mathfrak{s}^{i}} \mathfrak{r}^{k} \\
& +\frac{1}{2} \lim _{r \rightarrow \infty}\left[\left(\hat{\mathfrak{a}}^{-1}\right)^{l} \cdot \check{\mathfrak{a}}_{k}\right]\left(\tilde{Z}_{l i} \mathfrak{r}^{k}+\frac{\partial \mathfrak{r}^{k}}{\partial \mathfrak{s}^{i}} \tilde{Z}_{l j} \mathfrak{s}^{j}+\frac{\partial \mathfrak{r}^{j}}{\partial \mathfrak{s}^{i}} Z_{l j} \mathfrak{r}^{k}+\frac{\partial \mathfrak{r}^{k}}{\partial \mathfrak{s}^{i}} Z_{l j} \mathfrak{r}^{j}\right) .
\end{aligned}
$$

Here, we have omitted the arguments $k$ on the right-hand side. To obtain the final result, we observe that the third term on the right-hand side vanishes, since $z_{i j} \equiv 0$, as stated above. Moreover, the last term, which is the only one with a cutoff dependence, vanishes when the large-r limit is taken, because $\left(\widehat{\mathfrak{a}}^{-1}\right)^{l} \cdot \check{\mathfrak{a}}_{k}$ goes to zero. Hence, we end up with

$$
\left\langle\mathcal{O}_{i}\right\rangle_{1}=Z_{i j} \mathfrak{r}^{j}+\frac{1}{2} \tilde{Z}_{i j \mathfrak{s}^{j}}
$$

which holds both in momentum and position space. From (3.7), one obtains the connected two-point function

$$
\left\langle\mathcal{O}_{i}(x) \mathcal{O}_{j}(y)\right\rangle=Z_{i k}\left(-\square_{x}\right) \frac{\delta \mathfrak{r}^{k}(x)}{\delta \mathfrak{s}^{j}(y)}+\frac{1}{2} \widetilde{Z}_{i j}\left(-\square_{x}\right) \delta(x-y)
$$

As promised, (3.7) and (3.8) are finite in the limit $r_{0} \rightarrow \infty$, as the matrices $Z_{i j}$ and $\tilde{Z}_{i j}$ do not depend on $r$. Equation (3.7) agrees with (2.24) of [45], for which it provides the missing piece $Y_{i j}=Z_{i j}$ and identifies the contact term, which will be discussed further in Section 3.3.

In momentum space, (3.8) has a more practical form. Setting $y=0$ by translational invariance and Fourier transforming the coordinate $x$, one finds

$$
\int \mathrm{d}^{d} x \mathrm{e}^{i k x}\left\langle\mathcal{O}_{i}(x) \mathcal{O}_{j}(0)\right\rangle=Z_{i k}\left(k^{2}\right) \frac{\partial \mathfrak{r}^{k}}{\partial \mathfrak{s}^{j}}(k)+\frac{1}{2} \widetilde{Z}_{i j}\left(k^{2}\right) .
$$

In what follows, we will often work in momentum space omitting the argument $k$. By the two-point function $\left\langle\mathcal{O}_{i} \mathcal{O}_{j}\right\rangle$ in momentum space, we will intend (3.9).

Unfortunately, the symmetry of the two-point function under exchange of $\mathcal{O}_{i}$ and $\mathcal{O}_{j}$ is not obvious from (3.8). It would be a nontrivial test of any concrete calculation to see whether the right-hand side, with its antisymmetric second term, combines to something symmetric.

A different form of the 2-point function, which makes its pole structure more explicit, can be obtained with the help of the Green's function. The Green's function satisfies

$$
\left[\left(D_{r}+M-\frac{2 d}{d-1} W\right)\left(D_{r}-M\right)-\mathrm{e}^{-2 A} k^{2}\right] G\left(r, r^{\prime} ; k^{2}\right)=-\mathrm{e}^{-d A(r)} \delta\left(r-r^{\prime}\right),
$$


where the factor $\mathrm{e}^{-d A}$ on the right-hand side is the metric factor $1 / \sqrt{g}$ from the covariant delta function. The Green's function can be written in terms of a basis of eigenfunctions,

$$
G\left(r, r^{\prime}, k^{2}\right)=\sum_{\lambda} \frac{\mathfrak{a}_{\lambda}(r) \mathfrak{a}_{\lambda}\left(r^{\prime}\right)}{k^{2}+m_{\lambda}^{2}}
$$

where the functions $\mathfrak{a}_{\lambda}$ satisfy (2.49) for $k^{2}=-m_{\lambda}^{2}$. (Again, we omit the matrix indices, and the indices of the two $\mathfrak{a}_{\curlywedge}$ 's are not contracted.) Substituting (3.11) into (3.10) yields the completeness relation

$$
\sum_{\lambda} \mathfrak{a}_{\lambda}(r) \mathfrak{a}_{\lambda}\left(r^{\prime}\right)=\mathrm{e}^{-(d-2) A(r)} \delta\left(r-r^{\prime}\right),
$$

from which one can deduce the orthogonality relation

$$
\int \mathrm{d} r \mathrm{e}^{(d-2) A(r)} \mathfrak{a}_{\lambda}(r) \cdot \mathfrak{a}_{\sigma}(r)=\delta_{\lambda \sigma} .
$$

With the dot product we denote the covariant contraction of indices. Equation (3.13) provides the condition for the eigenstates $\mathfrak{a}_{\lambda}$ to be integrable. Due to the factor $\mathrm{e}^{(d-2) A}$, the integral measure is not the covariant bulk integral measure that one might have expected.

It can be checked in the various cases we consider that the asymptotically dominant behaviours, $\widehat{\mathfrak{a}}_{i}$, are not integrable, whereas the subdominant behaviours are integrable. Thus, we have

$$
\mathfrak{a}_{\lambda}(r)=\mathfrak{r}_{\lambda}^{i} \check{\mathfrak{a}}_{i}(r) .
$$

To derive a form of the 2-point function which makes its symmetry and pole structure manifest, we start by considering the general formula for a solution $\mathfrak{a}\left(r, k^{2}\right)$ of (2.49) in terms of the Green's function and prescribed boundary values. Let $r_{0}$ be a (large) cutoff parameter determining the hypersurface where the boundary values are formally prescribed. Remembering that neither the Green's function nor its derivative vanish at the cutoff boundary, we have (This formula follows from (3.10) upon multiplication by $\mathrm{e}^{d A(r)} \mathfrak{a}(r)$ from the left, taking the integral over $r$, integrating by parts and using the field equation (2.49). The IR boundary does not contribute, because $\mathrm{e}^{d A}$ vanishes there. The reason for this is that $r=r_{\text {IR }}$ should correspond to a single point of the bulk space, which is only guaranteed if $\mathrm{e}^{d A}$ vanishes there, c.f. (2.3).)

$$
\mathfrak{a}\left(r, k^{2}\right)=\mathrm{e}^{d A\left(r_{0}\right)}\left[\left(D_{r_{0}} \mathfrak{a}\left(r_{0}, k^{2}\right)\right) \cdot G\left(r_{0}, r ; k^{2}\right)-\mathfrak{a}\left(r_{0}, k^{2}\right) \cdot D_{r_{0}} G\left(r_{0}, r ; k^{2}\right)\right],
$$

where $\mathfrak{a}\left(r_{0}, k^{2}\right)$ and $D_{r_{0}} \mathfrak{a}\left(r_{0}, k^{2}\right)$ are the prescribed values of the field and its first derivative at the cutoff boundary, respectively. Since $r_{0}$ is an unphysical cutoff parameter, we must ensure 
that the bulk field $\mathfrak{a}(r)$ remains unchanged when $r_{0}$ is varied. This is easily achieved, if, together with a change of the cutoff, $r_{0} \rightarrow r_{0}+\delta r_{0}$, the boundary values are changed by

$$
\delta \mathfrak{a}\left(r_{0}, k^{2}\right)=\left(D_{r_{0}} \mathfrak{a}\left(r_{0}, k^{2}\right)\right) \delta r_{0}, \quad \delta D_{r_{0}} \mathfrak{a}\left(r_{0}, k^{2}\right)=\left(D_{r_{0}}^{2} \mathfrak{a}\left(r_{0}, k^{2}\right)\right) \delta r_{0}
$$

and the second derivative, $D_{r_{0}}^{2} \mathfrak{a}\left(r_{0}, k^{2}\right)$, is determined by the equation of motion (2.49). To assure (3.16), we determine the formal boundary values at the cutoff, $\mathfrak{a}\left(r_{0}, k^{2}\right)$ and $D_{r_{0}} \mathfrak{a}\left(r_{0}, k^{2}\right)$, from the generic asymptotic behaviour (2.54) (in momentum space), with coefficients $\mathfrak{r}^{i}$ and $\mathfrak{s}^{i}$ fixed. After inserting (2.54) and (3.11) into (3.15), we obtain

$$
\begin{aligned}
\mathfrak{a}\left(r, k^{2}\right)= & \mathrm{e}^{d A\left(r_{0}\right)} \sum_{\lambda} \frac{\mathfrak{a}_{\lambda}(r)}{k^{2}+m_{\lambda}^{2}} \\
& \times\left\{\mathfrak{r}^{l}\left[\left(D_{r_{0}} \check{\mathfrak{a}}_{l}\left(r_{0}, k^{2}\right)\right) \cdot \mathfrak{a}_{\lambda}\left(r_{0}\right)-\check{\mathfrak{a}}_{l}\left(r_{0}, k^{2}\right) \cdot D_{r_{0}} \mathfrak{a}_{\lambda}\left(r_{0}\right)\right]\right. \\
& \left.\quad+\mathfrak{s}^{l}\left[\left(D_{r_{0}} \widehat{\mathfrak{a}}_{l}\left(r_{0}, k^{2}\right)\right) \cdot \mathfrak{a}_{\lambda}\left(r_{0}\right)-\widehat{\mathfrak{a}}_{l}\left(r_{0}, k^{2}\right) \cdot D_{r_{0}} \mathfrak{a}_{\lambda}\left(r_{0}\right)\right]\right\} .
\end{aligned}
$$

To continue, we observe that for very large $r_{0}$, the term on the second line of (3.17), containing only subdominant solutions, is much smaller than the term on the third line. Therefore, we drop it. Moreover, as we are interested only in the pole structure, we consider $k^{2}$ very close to $-m_{\lambda}^{2}$ and expand the numerator keeping only the leading term, that is, we replace $k^{2}$ by $-m_{\lambda}^{2}$ in the numerator. Finally, we use the fact that the eigenfunctions are purely subdominant, (At this point one may wonder where the dominant part of $\mathfrak{a}$ comes from. It arises from the sum over the spectrum in (3.17), in particular from the UV contribution. For the simple case of AdS bulk, this is shown in appendix A.1 of [45]. However, it does not contribute to the poles.)

$$
\mathfrak{a}_{\lambda}(r)=\mathfrak{r}_{\lambda}^{i} \check{\mathfrak{a}}_{i}\left(r,-m_{\lambda}^{2}\right) .
$$

This yields

$$
\mathfrak{a}\left(r, k^{2}\right) \underset{k^{2} \approx-m_{\lambda}^{2}}{=} \mathfrak{s}^{l} Z_{l j}\left(-m_{\lambda}^{2}\right) \frac{\mathfrak{r}_{\lambda}^{j} \mathfrak{r}_{\lambda}^{i}}{k^{2}+m_{\lambda}^{2}} \check{\mathfrak{a}}_{i}\left(r,-m_{\lambda}^{2}\right),
$$

with $Z_{i j}$ defined in (3.4).

Thus, after reading off the response function $\mathfrak{r}^{i}$ (for $k^{2} \approx-m_{\lambda}^{2}$ ) from (3.19), we obtain the poles of the connected 2-point function, using (3.7) and differentiating with respect to the source $\mathfrak{s}^{j}$,

$$
\left\langle\mathcal{O}_{i}(k) \mathcal{O}_{j}(-k)\right\rangle=\sum_{\lambda} Z_{i i^{\prime}}\left(-m_{\lambda}^{2}\right) Z_{j j^{\prime}}\left(-m_{\lambda}^{2}\right) \frac{\mathfrak{r}_{\lambda}^{i^{\prime}} \mathfrak{r}_{\lambda}^{j^{\prime}}}{k^{2}+m_{\lambda}^{2}}+\text { c.t.. }
$$


Therefore, defining also

$$
\begin{aligned}
Z_{\lambda, i} & =Z_{i i^{\prime}}\left(-m_{\lambda}^{2}\right) \mathfrak{r}_{\lambda}^{i^{\prime}} \\
& =\mathrm{e}^{d A(r)}\left[\left(D_{r} \widehat{\mathfrak{a}}_{i}\left(r,-m_{\lambda}^{2}\right)\right) \cdot \mathfrak{a}_{\lambda}(r)-\widehat{\mathfrak{a}}_{i}\left(r,-m_{\lambda}^{2}\right) \cdot D_{r} \mathfrak{a}_{\lambda}(r)\right],
\end{aligned}
$$

our final result is

$$
\left\langle\mathcal{O}_{i}(k) \mathcal{O}_{j}(-k)\right\rangle=\sum_{\lambda} \frac{Z_{\lambda, i} Z_{\lambda, j}}{k^{2}+m_{\lambda}^{2}}+\text { c.t. }
$$

We note that the $Z_{\lambda, i}$ are independent of the choice of the subdominant basis solutions, because the normalization of the eigenfunctions $\mathfrak{a}_{\lambda}$ is fixed by (3.13). Moreover, (3.22) shows that the pole terms of the 2-point functions are manifestly symmetric under exchange of $\mathcal{O}_{i}$ and $\mathcal{O}_{j}$.

\subsection{VEVs}

Let us make a few comments on VEVs. Equation (3.5) only gives the part of the one-point function which is linear in the fluctuations. At the moment, our approach does not allow for a systematic derivation of the VEVs yet. However, we would like to make the following observation. The scalar equations (2.49) have the zero mode solution $\left(k^{2}=0\right)$

$$
\overline{\mathfrak{a}}^{a} \sim \frac{W^{a}}{W}
$$

which only depends on the radial variable $r$. Like any fluctuation, this has an expansion into dominant and subdominant asymptotic solutions as in (2.54). In aAdS settings, nonzero coefficients of the dominant and subdominant basis solutions are interpreted as finite couplings and VEVs, respectively. As mentioned in the introduction, the interpretation of finite couplings (in the sense of a deformation of some special QFT) cannot be made in the general case, but we will continue to interpret the presence of asymptotically subdominant behaviour as finite VEVs. However, this just means that the VEV is nonzero, because the normalization factor in (3.23) is undetermined. In addition, there is an issue of scheme dependence, when nonzero dominant behaviours are present, but this happens also in aAdS settings, as will be seen in Sections 4 and 5 .

\subsection{Scheme Dependence}

In QFT, contact terms of correlation functions, which do not influence physical scattering amplitudes, depend on the renormalization scheme. Let us now discuss how the scheme dependence of the two-point functions (3.8) appears from a bulk point of view. For the sake of brevity, we will work in momentum space and omit all functional arguments.

The starting point is the decomposition (2.54) of a regular solution to the bulk field equations. Clearly, the definition of the asymptotic solutions $\widehat{\mathfrak{a}}_{i}$ and $\check{\mathfrak{a}}_{i}$ is not unique. Our 
restriction on the functional form of these solutions in terms of series of $k^{2}$ and the fact that all subdominant solutions are negligible for large $r$ with respect to all dominant ones still allows for a change of basis of the form

$$
\widehat{\mathfrak{a}}_{i}^{\prime}=\Lambda_{i}^{j} \widehat{\mathfrak{a}}_{j}+\lambda_{i}^{j} \check{\mathfrak{a}}_{j}, \quad \check{\mathfrak{a}}_{i}^{\prime}=\mu_{i}^{j} \check{\mathfrak{a}}_{j},
$$

where the (nondegenerate) matrices $\Lambda_{i j}, \lambda_{i j}$ and $\mu_{i j}$ are polynomials in $k^{2}$. Under this change of basis, the matrices $\tilde{Z}_{i j}$ and $Z_{i j}$ transform into (Remember $z_{i j}=0$.)

$$
\begin{gathered}
\tilde{Z}_{i j}^{\prime}=\Lambda_{i}^{k} \Lambda_{j}^{l} \tilde{Z}_{k l}+\left(\Lambda_{i}^{k} \lambda_{j}^{l}-\Lambda_{i}^{k} \lambda_{j}^{l}\right) Z_{k l}, \\
Z_{i j}^{\prime}=\Lambda_{i}^{k} \mu_{j}^{l} Z_{k l},
\end{gathered}
$$

respectively, while the source and response coefficients in the new basis become

$$
\mathfrak{s}^{\prime i}=\mathfrak{s}^{j}\left(\Lambda^{-1}\right)_{j}^{i}, \quad \mathfrak{r}^{\prime i}=\left[\mathfrak{r}^{j}-\mathfrak{s}^{l}\left(\Lambda^{-1}\right)_{l}^{k} \lambda_{k}^{j}\right]\left(\mu^{-1}\right)_{j}^{i} .
$$

Inserting these transformations into (3.8), one finds the connected two-point functions of the operators $\mathcal{O}_{i}^{\prime}$ coupling to the sources $\mathfrak{s}^{\prime i}$,

$$
\left\langle\mathcal{O}_{i}^{\prime} \mathcal{O}_{j}^{\prime}\right\rangle=\Lambda_{i}^{k} \Lambda_{j}^{l}\left\langle\mathcal{O}_{k} \mathcal{O}_{l}\right\rangle-\frac{1}{2}\left(\Lambda_{i}^{k} \lambda_{j}^{l}+\Lambda_{j}^{k} \lambda_{i}^{l}\right) Z_{k l}
$$

Hence, the matrix $\Lambda_{i}^{j}$ performs a rotation of the basis of operators, as one would have expected, while a nonzero $\lambda_{i}^{j}$ changes the contact terms, which corresponds to a change of renormalization scheme.

In QFT, operators are usually characterized by their scaling dimension, which is renormalization scale dependent. Under renormalization, they undergo operator mixing, such that an operator of a given dimension, defined at a certain renormalization scale, is generically made up of the operators of equal and lower dimensions, defined at a larger renormalization scale. There is, however, some ambiguity, as operators of equal dimension and otherwise equal quantum numbers can be arbitrarily combined to equivalent combinations. This ambiguity finds a natural counterpart in the present approach. Ordering the dominant asymptotic solutions according to their asymptotic behaviour in descending order, it is natural to choose $\Lambda$ in "upper triangular" form, such that each dominant solution is modified only by solutions of equal and weaker asymptotic behaviour. A similar remark would apply for $\mu$.

A further restriction on the redefinition could come from the fact that the lowest order terms in a near boundary expansion of the dominant solutions typically have a definite correlation between powers of $\mathrm{e}^{-r}$ and powers of $k^{2}$. This is well known, for instance, in the aAdS case with a single scalar field, compare for example, the discussion in Section 5.1 of [12]. Something similar happens in the case of KS, as can be seen from the explicit form of the asymptotic solutions given in the appendix of [29] (we refrain from reproducing them here as they are very "bulky"). We will refer to a choice of dominant solutions respecting this correlation as a "natural" choice. 
Finally, we remark that it is reasonable to assume that $\Lambda_{i}^{j}$ and /or $\mu_{i}^{j}$ can be chosen such that $Z_{i j}^{\prime}=\delta_{i j}$ in (3.25). A possible obstruction to this possibility would be that the matrices needed to achieve that are nonpolynomial in $k^{2}$. We will see later that the choice $Z_{i j}=\delta_{i j}$ is possible for the KS system. Starting with such a choice, a further change of basis using just $\lambda_{i j}$ would lead to

$$
\tilde{Z}_{i j}^{\prime}=\tilde{Z}_{i j}+\lambda_{j i}-\lambda_{i j}
$$

implying that one can achieve $\tilde{Z}_{i j}^{\prime}=0$ by a suitable choice of $\lambda_{i j}$, although this choice is obviously not unique.

\section{Examples}

In this section, we will compare the general expressions from the previous sections with the results of holographic renormalization in the case of aAdS bulk space-times. To carry out HR, one can use one's favourite method from the choice of $[10,11,14]$. We will start with pure AdS and then consider some favourite RG flows, the GPPZ [56] and the Coulomb branch flows $[57,58]$.

\subsection{Scalars in AdS Background}

As the simplest case, we consider a number of free massive scalars in a pure AdS background. The results are, of course, dictated by conformal invariance of the boundary CFT, but it is still useful to deal with this case, because several statements that we make in what follows hold in any aAdS configuration. In this and the following subsections we will set the AdS length scale to $L=1$. It can be reinstated by dimensional analysis.

An AdS background exists for any superpotential with a fixed point (where $W$ is nonvanishing). We consider the scalars parametrized by Riemann normal coordinates around the fixed point and their local coordinate system rotated in such a way that the matrix of second derivatives of $W$ is diagonal [33]. This gives rise to a set of canonical scalar fields with a superpotential

$$
W(\phi)=-\frac{d-1}{2}+\frac{1}{2} \sum_{a} w_{a}\left(\phi^{a}\right)^{2}+\mathcal{O}\left(\phi^{3}\right) .
$$

The $(d+1)$-dimensional AdS background metric is given by

$$
\mathrm{d} s^{2}=\frac{1}{z^{2}}\left(\mathrm{~d} z^{2}+\eta_{\mu \nu} \mathrm{d} x^{\mu} \mathrm{d} x^{\nu}\right)
$$

where the radial variable $z$ is related to $r$ of Section 2 by $z=\mathrm{e}^{-r}$. To linear order, the scalar fields satisfy the equations of motion

$$
\left(\partial_{z}^{2}-\frac{d-1}{z} \partial_{z}-\frac{m_{a}^{2}}{z^{2}}-k^{2}\right) \mathfrak{a}^{a}=0
$$


where no summation over $a$ is intended. The masses are related to the coefficients $w_{a}$ of (4.1) by

$$
m_{a}^{2}=w_{a}^{2}+d w_{a}
$$

\section{Z Matrices}

For Re $k \geq 0$, the conventionally normalized asymptotic solutions of (4.3) are

$$
\begin{aligned}
& \widehat{\mathfrak{a}}_{i}^{a}(z, k)=\delta_{i}^{a} \Gamma\left(1-\alpha_{a}\right)\left(\frac{k}{2}\right)^{\alpha_{a}} z^{d / 2} I_{-\alpha_{a}}(k z)=\delta_{i}^{a} z^{d / 2-\alpha_{a}}+\cdots, \\
& \check{\mathfrak{a}}_{i}^{a}(z, k)=\delta_{i}^{a} \Gamma\left(1+\alpha_{i}\right)\left(\frac{k}{2}\right)^{-\alpha_{a}} z^{d / 2} I_{\alpha_{a}}(k z)=\delta_{i}^{a} z^{d / 2+\alpha_{a}}+\cdots,
\end{aligned}
$$

(no summation over $a$ ) where the $I_{\alpha}$ are modified Bessel functions, and

$$
\alpha_{a}=\sqrt{\frac{d^{2}}{4}+m_{a}^{2}} .
$$

The powers of $k$ in front of the solutions are necessary in order to make the leading terms $k$-independent. It is straightforward to verify that the matrices (3.4) are (as the solutions are diagonal, that is, proportional to $\delta_{i}^{a}$, we use $\alpha_{a}$ and $\alpha_{i}$ interchangeably)

$$
Z_{i j}=2 \alpha_{i} \delta_{i j}, \quad \tilde{Z}_{i j}=0, \quad z_{i j}=0 .
$$

These equalities hold true in any aAds configuration, as long as one uses the same asymptotic normalizations.

\section{Spectrum}

Equation (4.3) admits a continuous spectrum of regular and subdominant solutions for $k^{2}=$ $-\lambda^{2}, \lambda>0$. The eigenfunctions are given by (The generic label $\lambda$ for the eigenfunctions used in Section 3 [c.f. (3.11)] is replaced here by two indices, $\lambda=-k^{2}$ and $i=1, \ldots, n$. As before, the upper index $a$ is the vector component index.)

$$
\mathfrak{a}_{\lambda i}^{a}(z)=\delta_{i}^{a} \sqrt{\lambda} z^{d / 2} \mathrm{~J}_{\alpha_{i}}(\lambda z)
$$

where the $\mathrm{J}_{\alpha}$ are Bessel functions, and they satisfy the orthogonality relation

$$
\int_{0}^{\infty} \frac{\mathrm{d} z}{z} z^{-(d-2)} \mathfrak{a}_{\lambda i}(z) \cdot \mathfrak{a}_{\lambda^{\prime} j}(z)=\delta\left(\lambda-\lambda^{\prime}\right) \delta_{i j}
$$


Considering the small- $z$ behaviour of (4.9) and comparing with (4.6), one can read off the response coefficients $\mathfrak{r}_{\lambda}^{i}$ of the eigenfunctions, compare for example, (3.18),

$$
\mathfrak{r}_{\lambda}^{i}=\left(\frac{\lambda}{2}\right)^{\alpha_{i}} \frac{\sqrt{\lambda}}{\Gamma\left(1+\alpha_{i}\right)} .
$$

Hence, after using (3.21), one obtains the two-point function in the form (3.22) as the sum (here it is an integral) over the spectrum

$$
\left\langle\mathcal{O}_{i}(k) \mathcal{O}_{j}(-k)\right\rangle=\delta_{i j} \frac{2^{2\left(1-\alpha_{i}\right)}}{\Gamma\left(\alpha_{i}\right)^{2}} \int_{0}^{\infty} \mathrm{d} \lambda \frac{\lambda^{2 \alpha_{i}+1}}{k^{2}+\lambda^{2}}=-\delta_{i j} \frac{2 \Gamma\left(1-\alpha_{i}\right)}{\Gamma\left(\alpha_{i}\right)}\left(\frac{k}{2}\right)^{2 \alpha_{i}} .
$$

Notice that the second equality holds only after an analytic continuation, because the integral does not exist if $\alpha_{i} \geq 0$. This is equivalent to adding an infinite contact term to the integral over the spectrum. For example, if $0<\alpha_{i}<1$, we rewrite the integrand as

$$
\frac{\lambda^{2 \alpha_{i}+1}}{k^{2}+\lambda^{2}}=\lambda^{2 \alpha_{i}-1}-k^{2} \frac{\lambda^{2 \alpha_{i}-1}}{k^{2}+\lambda^{2}}
$$

and add a contact term that cancels the first term on the right-hand side.

\subsection{GPPZ Flow}

In the GPPZ flow [56], we consider two canonical scalar fields $\phi$ and $\sigma$ with the superpotential

$$
W(\phi)=-\frac{3}{4}\left[\cosh \frac{2 \phi}{\sqrt{3}}+\cosh (2 \sigma)\right]
$$

Both are dual to operators with bare dimensions $\Delta=3$. The scalar $\phi$ is the active scalar of the GPPZ flow, that is, it is nontrivial in the background. More relations for the GPPZ flow can be found in $[65,66]$.

\section{Counterterms and Scheme Dependence}

Let us start by reviewing the treatment in standard AdS/CFT [10, 11, 14]. Being dual to operators of dimension 3 , the scalar fields behave asymptotically as

$$
\begin{aligned}
& \phi(r)=\phi_{0} \mathrm{e}^{-r}+\psi_{2} r \mathrm{e}^{-3 r}+\phi_{2} \mathrm{e}^{-3 r}+\cdots, \\
& \sigma(r)=\sigma_{0} \mathrm{e}^{-r}+\rho_{2} r \mathrm{e}^{-3 r}+\sigma_{2} \mathrm{e}^{-3 r}+\cdots,
\end{aligned}
$$

where $\phi_{0}, \phi_{2}, \sigma_{0}$, and $\sigma_{2}$ are independent coefficients, whereas $\psi_{2}$ and $\rho_{2}$ depend on $\phi_{0}$ and $\sigma_{0}$. The background solution satisfies

$$
\mathrm{e}^{2 \bar{\phi} / \sqrt{3}}=\frac{1+\mathrm{e}^{-r}}{1-\mathrm{e}^{-r}}, \quad \bar{\sigma}=0
$$


which implies

$$
\bar{\phi}_{0}=\sqrt{3}, \quad \bar{\psi}_{2}=0, \quad \bar{\phi}_{2}=\frac{1}{\sqrt{3}} .
$$

Carrying out holographic renormalization $[10,11,14]$, one finds the coefficients $\psi_{2}$ and $\rho_{2}$

$$
\begin{gathered}
\psi_{2}=-\frac{1}{2} k^{2} \phi_{0}+\frac{4}{3} \sigma_{0}^{2} \phi_{0} \\
\rho_{2}=-\frac{1}{2} k^{2} \sigma_{0}-\frac{8}{3} \sigma_{0}^{3}+\frac{4}{3} \phi_{0}^{2} \sigma_{0}
\end{gathered}
$$

as well as the exact one-point functions with the full scheme dependence, (We have omitted the curvature-dependent terms, which are irrelevant here.)

$$
\begin{gathered}
\left\langle\mathcal{O}_{\phi}\right\rangle=2 \phi_{2}+\left(\frac{1}{2}+m_{0}\right) k^{2} \phi_{0}+\frac{1}{6} u_{4 \phi} \phi_{0}^{3}+\left(\frac{1}{2} u_{4 \phi \sigma}-\frac{4}{3}\right) \sigma_{0}^{2} \phi_{0} \\
\left\langle\mathcal{O}_{\sigma}\right\rangle=2 \sigma_{2}+\left(\frac{1}{2}+\tilde{m}_{0}\right) k^{2} \sigma_{0}+\left(\frac{1}{6} u_{4 \sigma}+\frac{8}{3}\right) \sigma_{0}^{3}+\left(\frac{1}{2} u_{4 \phi \sigma}-\frac{4}{3}\right) \phi_{0}^{2} \sigma_{0} .
\end{gathered}
$$

The scheme-dependent coefficients that appear here stem from the addition of finite counterterms of the form

$$
g^{i j}\left(\partial_{i} \phi\right)\left(\partial_{j} \phi\right), \quad g^{i j}\left(\partial_{i} \sigma\right)\left(\partial_{j} \sigma\right), \quad \phi^{4}, \quad \phi^{2} \sigma^{2}, \quad \sigma^{4}
$$

Let us linearize around the background (4.16), (4.17), which is sufficient to extract the information for the two-point functions, and translate the fluctuation into the gaugeinvariant variables. One must take some care for the active scalar. Its mixing with the metric fluctuations is described by (2.22), (2.23), and (2.45), where we must set $v=0$, because of the orthogonal gauge used to derive (4.19). Hence, using

$$
\mathfrak{a}^{\phi}=\varphi^{\phi}+\frac{W^{\phi}}{4 W} h, \quad \mathfrak{a}^{\sigma}=\varphi^{\sigma}, \quad \mathfrak{b}=\partial_{r}\left(\frac{h}{4 W}\right)=-\frac{W_{\phi}}{W} \mathfrak{a}^{\phi},
$$

one arrives at

$$
\begin{gathered}
\left\langle\mathcal{O}_{\phi}\right\rangle=\frac{\sqrt{3}}{2}\left(u_{4 \phi}+\frac{4}{3}\right)+\left[2 \mathfrak{a}_{2}^{\phi}+\frac{3}{2}\left(u_{4 \phi}+\frac{4}{3}\right) \mathfrak{a}_{0}^{\phi}+\left(m_{0}+\frac{1}{2}\right) k^{2} \mathfrak{a}_{0}^{\phi}\right] \\
\left\langle\mathcal{O}_{\sigma}\right\rangle=2 \mathfrak{a}_{2}^{\sigma}+\left(\tilde{m}_{0}+\frac{1}{2}\right) k^{2} \mathfrak{a}_{0}^{\sigma}+\left(\frac{3}{2} u_{4 \phi \sigma}-4\right) \mathfrak{a}_{0}^{\sigma} .
\end{gathered}
$$

The coefficients $\mathfrak{a}_{0}, \mathfrak{a}_{2}$ with superscripts $\phi$ and $\sigma$ are the independent coefficients of the asymptotic expansion, that is, the analogue of (4.15), of the components of $\mathfrak{a}$. The first term on 
the right-hand side of (4.22) is a scheme-dependent, that is, unphysical, VEV, which vanishes in renormalization schemes that respect SUSY $\left(u_{4 \phi}=-4 / 3\right)$ [12].

Let us now compare these expressions with the results of Section 3.1. In particular, let us define the dominant and subdominant solutions by

$$
\begin{gathered}
\widehat{\mathfrak{a}}^{\phi}=\mathrm{e}^{-r}-r \mathrm{e}^{-3 r} \frac{1}{2} k^{2}+\mathrm{e}^{-3 r} c^{\phi}+\cdots, \quad \check{\mathfrak{a}}^{\phi}=\mathrm{e}^{-3 r}+\cdots, \\
\widehat{\mathfrak{a}}^{\sigma}=\mathrm{e}^{-r}-r \mathrm{e}^{-3 r} \frac{1}{2}\left(k^{2}-8\right)+\mathrm{e}^{-3 r} c^{\sigma}+\cdots, \quad \check{\mathfrak{a}}^{\sigma}=\mathrm{e}^{-3 r}+\cdots,
\end{gathered}
$$

with two as yet undetermined coefficients $c^{\phi}$ and $c^{\sigma}$. The second terms in the dominant solution can be determined directly from (4.18). From (4.23), one obtains the counterterm matrix (3.3)

$$
\begin{gathered}
U_{\phi \phi}=-r \mathrm{e}^{-2 r} k^{2}+\mathrm{e}^{-2 r}\left(\frac{1}{2} k^{2}+2 c^{\phi}\right)+\cdots, \\
U_{\sigma \sigma}=-2-r \mathrm{e}^{-2 r}\left(k^{2}-8\right)+\mathrm{e}^{-2 r}\left(\frac{1}{2} k^{2}-4+2 c^{\sigma}\right)+\cdots, \\
U_{\phi \sigma}=U_{\sigma \phi}=0,
\end{gathered}
$$

where the ellipses indicate terms which do not contribute and can be truncated. It can be noted that the resulting divergent counterterms agree with the divergent counterterms from the standard approach. The last terms shown give finite contributions.

It is straightforward to verify that the $Z$ matrices (3.4) that one obtains from (4.23) agree with the AdS result (4.8).

Writing the bulk field $\mathfrak{a}$ in terms of the asymptotic solutions (4.23) gives the source and response coefficients, (the expressions for $\phi$ and $\sigma$ are identical)

$$
\mathfrak{s}=\mathfrak{a}_{0}, \quad \mathfrak{r}=\mathfrak{a}_{2}-c \mathfrak{a}_{0},
$$

so that the linear term of the one-point function (3.7) becomes

$$
\langle\mathcal{O}\rangle_{1}=2\left(\mathfrak{a}_{2}-c \mathfrak{a}_{0}\right)
$$

Comparing this with the linear terms in (4.22), we find agreement in the nonlocal term containing $\mathfrak{a}_{2}$. Moreover, we can determine the constants $c^{\phi}$ and $c^{\sigma}$ as (An alternative way of determining them is to compare the finite counterterms that result from (4.24) with the linearized standard ones.)

$$
c^{\phi}=-\frac{3}{4}\left(u_{4 \phi}+\frac{4}{3}\right)-\frac{1}{4}\left(2 m_{0}+1\right) k^{2}, \quad c^{\sigma}=-\left(\frac{3}{4} u_{4 \phi \sigma}-2\right)-\frac{1}{4}\left(2 \tilde{m}_{0}+1\right) k^{2} .
$$

This result states explicitly the relation between the choice of the dominant basis and the renormalization scheme. Note that only $c^{\phi}, c^{\sigma} \sim\left(k^{2}\right)^{n}$ for $n=0$ or 1 appear. 
Spectrum of the Active Scalar

Let us illustrate formula (3.22), which represents the 2-point function as a sum over its poles, up to local terms, using as an example the active GPPZ scalar. The gauge invariant equation of motion reads $[31,66]$

$$
\left[u(1-u) \partial_{u}^{2}-2(1-u) \partial_{u}-\frac{k^{2}}{4}\right] \frac{\mathfrak{a}^{\phi}}{\sqrt{1-u}}=0
$$

where the radial coordinate $u$ is defined by $u=1-\mathrm{e}^{-2 r}$, and the warp factor is $\mathrm{e}^{2 A}=u /(1-u)$.

Equation (4.28) admits a discrete spectrum of regular and subdominant eigenfunctions, with mass squares

$$
m_{n}^{2}=4 n(n+1), \quad n=1,2,3, \ldots
$$

The normalized eigenfunctions are

$$
\mathfrak{a}_{n}^{\phi}(u)=\sqrt{\frac{2(2 n+1)}{n(n+1)}}(1-u)^{3 / 2} \frac{\mathrm{d}}{\mathrm{d} u} P_{n}(2 u-1),
$$

where $P_{n}$ are Legendre polynomials. (As in $[31,66]$, regular and subdominant solutions of (4.28) are given by Jacobi polynomials $P_{n-1}^{(1,1)}(z)$, which are proportional to $\mathrm{d} P_{n}(z) / \mathrm{d} z$.) One easily finds the response coefficients

$$
\mathfrak{r}_{n}^{\phi}=\sqrt{2 n(n+1)(2 n+1)} .
$$

Thus, we obtain for the 2-point function (3.22),

$$
\left\langle\mathcal{O}_{\phi}(k) \mathcal{O}_{\phi}(-k)\right\rangle=\sum_{n=1}^{\infty} \frac{8 n(n+1)(2 n+1)}{k^{2}+4 n(n+1)}+\text { c.t.. }
$$

Clearly, the sum in (4.32) does not converge, so that there are again infinite contact terms. It is instructive to compare (4.32) with the finite result from holographic renormalization [61,67]. Let us pick the particular SUSY scheme $u_{4 \phi}=-4 / 3, m_{0}=-1 / 2$. Then

$$
\left\langle\mathcal{O}_{\phi}(k) \mathcal{O}_{\phi}(-k)\right\rangle=\frac{k^{2}}{2}\left[\psi\left(\frac{3+\sqrt{1-k^{2}}}{2}\right)+\psi\left(\frac{3-\sqrt{1-k^{2}}}{2}\right)-\psi(1)-\psi(2)\right],
$$

where $\psi(z)=[\ln \Gamma(z)]^{\prime}$. Using the identity

$$
\psi(x)-\psi(y)=\sum_{n=0}^{\infty}\left(\frac{1}{y+n}-\frac{1}{x+n}\right)
$$


we obtain from (4.33)

$$
\begin{aligned}
\left\langle\mathcal{O}_{\phi}(k) \mathcal{O}_{\phi}(-k)\right\rangle & =\frac{k^{4}}{2} \sum_{n=1}^{\infty} \frac{2 n+1}{n(n+1)\left[k^{2}+4 n(n+1)\right]} \\
& =\sum_{n=1}^{\infty}\left[\frac{8 n(n+1)(2 n+1)}{k^{2}+4 n(n+1)}-2(2 n+1)+k^{2} \frac{2 n+1}{2 n(n+1)}\right] .
\end{aligned}
$$

The nonlocal part agrees precisely with (4.32), and the scheme-dependent terms in (4.22) have the same form as the infinite contact terms.

\subsection{Coulomb Branch Flow}

Let us consider the Coulomb branch (CB) flow $[57,58]$. There is a canonical bulk scalar with the superpotential

$$
W(\phi)=-\mathrm{e}^{-2 \phi / \sqrt{6}}-\frac{1}{2} \mathrm{e}^{4 \phi / \sqrt{6}}
$$

The background solution is given by the relations

$$
v=\mathrm{e}^{\sqrt{6} \bar{\phi}}, \quad \mathrm{e}^{2 A}=l^{2} \frac{v^{2 / 3}}{1-v^{\prime}}
$$

introducing the radial variable $v$. The length $l$ is independent of the AdS radius $L(=1)$, together with which it determines the radius of the disc on which the D3 branes are distributed $\left(l^{2} / L\right)$.

\section{Counterterms and Scheme Dependence}

Let us again briefly review the asymptotic analysis form standard AdS/CFT. It follows from (4.36) that $\phi$ is dual to an operator of bare dimension $\Delta=2$. Correspondingly, it has an asymptotic expansion of the special form

$$
\phi(r)=\phi_{0} r \mathrm{e}^{-2 r}+\tilde{\phi}_{0} \mathrm{e}^{-2 r}+\cdots,
$$

where $\phi_{0}$ and $\tilde{\phi}_{0}$ are the two independent coefficients. Asymptotically, the background vanishes at the rate

$$
\bar{\phi} \approx-\frac{l^{2}}{\sqrt{6}} \mathrm{e}^{-2 r}
$$

implying

$$
\bar{\phi}_{0}=0, \quad \overline{\tilde{\phi}}_{0}=-\frac{l^{2}}{\sqrt{6}} .
$$


From HR, the exact one-point function of the corresponding operator is given by [10, $11,67]$

$$
\langle\mathcal{O}\rangle=\tilde{\phi}_{0}+u_{2} \phi_{0}
$$

Two comments are in order here. The second term, involving a scheme-dependent constant $u_{2}$, is new compared to the corresponding formulas of $[10,11,67]$ and arises from the addition of a finite counterterm proportional to

$$
\left.\frac{\phi^{2}}{r^{2}}\right|_{r=r_{0}}
$$

Furthermore, our result differs from $[10,67]$ by a factor of $1 / 2$, as our $\phi_{0}$ differs by a factor -2 from theirs and our definition of the one-point function (1.2) exhibits an additional minus sign.

In contrast to the GPPZ flow, there is a scheme-independent VEV,

$$
\langle\mathcal{O}\rangle_{0}=-\frac{l^{2}}{\sqrt{6}}
$$

The presence of this VEV (but not its value) can also be inferred from the background mode,

$$
\frac{W^{\phi}}{W}=-\frac{4}{\sqrt{6}} \frac{1-v}{2+v}=-\frac{4}{3 \sqrt{6}} l^{2} \mathrm{e}^{-2 r}+\cdots
$$

Linearizing around the background and switching to the gauge invariant scalar, with an asymptotic expansion

$$
\mathfrak{a}=\mathfrak{a}_{0} r \mathrm{e}^{-2 r}+\tilde{\mathfrak{a}}_{0} \mathrm{e}^{-2 r}+\cdots,
$$

the one-point function (4.41) reads

$$
\langle\mathcal{O}\rangle=-\frac{l^{2}}{\sqrt{6}}+\tilde{\mathfrak{a}}_{0}+u_{2} \mathfrak{a}_{0}
$$

In order to make contact to Section 3.1, let us define the dominant and subdominant solutions as

$$
\widehat{\mathfrak{a}}=r \mathrm{e}^{-2 r}+\tilde{\alpha} \mathrm{e}^{-2 r}, \quad \check{\mathfrak{a}}=\mathrm{e}^{-2 r} .
$$

Writing (4.45) in this basis, one can read off the source and response as

$$
\mathfrak{s}=\mathfrak{a}_{0}, \quad \mathfrak{r}=\tilde{\mathfrak{a}}_{0}-\tilde{\alpha} \mathfrak{a}_{0} .
$$


In this case, (3.4) results in $\tilde{Z}=0$ and $Z=1$. (The case $\Delta=2$ would imply $\alpha=0$. The generic (4.8) does not apply, because of the logarithm in the dominant solution.) Thus, (3.7) reads

$$
\langle\mathcal{O}\rangle_{1}=\tilde{\mathfrak{a}}_{0}-\tilde{\alpha} \mathfrak{a}_{0} .
$$

Comparison with the part of (4.46) that is linear in the fluctuation implies $\tilde{\alpha}=-u_{2}$.

Finally, the counterterm "matrix" (3.3) obtained from the basis (4.47) is

$$
U=-\frac{1}{r}+\frac{\tilde{\alpha}}{r^{2}}+\mathcal{O}\left(r^{-3}\right)
$$

This provides the standard logarithmically divergent counterterm and a scheme-dependent finite contribution.

\section{Two-Point Function and Spectrum}

The two-point function for the CB flow was calculated in [61, 67]. Here, we present the calculation using the equation of motion in the gauge-invariant formalism. To obtain the twopoint function of the scalar operator dual to $\mathfrak{a}$, consider the equation of motion (2.49), which can be written in the form

$$
\begin{aligned}
& \left\{v\left[v(1-v) \partial_{v}^{2}+(2 \alpha-(2 \alpha-1) v) \partial_{v}-(\alpha-1)^{2}\right]\right. \\
& \left.\quad+2\left[v(1-v) \partial_{v}^{2}+(2 \alpha-(2 \alpha+2) v) \partial_{v}-(\alpha-1)(\alpha+2)\right]\right\} \frac{2+v}{1-v} v^{1-\alpha} \mathfrak{a}=0,
\end{aligned}
$$

where $\alpha=\left(1+\sqrt{1+k^{2}}\right) / 2$. For convenience, we have set also $l=1$. The regular solution of (4.51), up to an irrelevant normalization, is found to be

$$
\mathfrak{a}=B(\alpha-1, \alpha+1) v^{\alpha-1} \frac{1-v}{2+v}[F(\alpha-1, \alpha-1 ; 2 \alpha ; v)-3 F(\alpha-1, \alpha+1 ; 2 \alpha ; v)]
$$

where $B(x, y)=\Gamma(x) \Gamma(y) / \Gamma(x+y)$. From this solution one can read off the two-point function $[61,67]$ (shown for the scheme $u_{2}=0$ )

$$
\langle\mathcal{O}(k) \mathcal{O}(-k)\rangle=-\psi(\alpha)+\psi(1)+\frac{4}{3 k^{2}} .
$$

The spectrum is continuous for all $k^{2}<-1$, with an isolated pole at $k^{2}=0$ due to a Goldstone mode. The spectral density for $m^{2}=-k^{2}$ is found to be [4]

$$
\rho(m)=-\left.2 \Im\langle\mathcal{I}(k) \mathcal{O}(-k)\rangle\right|_{k^{2}=-m^{2}+i \epsilon}=\pi \tanh \left(\frac{\pi}{2} \sqrt{m^{2}-1}\right)+\frac{8}{3} \pi \delta\left(m^{2}\right) .
$$




\section{KS System}

Finally, we would like to apply the general discussion of Section 3 to the case of the KlebanovStrassler theory. We start by reviewing the relevant facts about the background.

\subsection{KS Background}

The effective $5 \mathrm{~d}$ model describing the bulk dynamics of the KS system contains seven scalar fields. (As in $[33,45]$, we restrict ourselves to the $J^{P C}=0^{++}$scalar sector, where $C$ denotes the quantum number under the $\mathbb{Z}_{2}$ charge conjugation symmetry of the KS theory, compare for example, $[68,69]$. Additional scalar fluctuations with $J^{P C}=0^{+-}$and $J^{P C}=0^{--}$were discussed in $[46,68,69]$.) We will use the Papadopoulos-Tseytlin [36] variables $\left(x, p, y, \Phi, b, h_{1}, h_{2}\right)$. The dual operators have dimensions $\Delta=8,7,6$ and twice 4 and 3 each. (It is not obvious that this is a meaningful statement, because in contrast to aAdS settings, the KS system has no UV conformal fixed point, where the operator dimensions can be fixed. However, the deviation from aAdS behaviour is quite mild, such that the asymptotic solutions behave nearly as if the dual operators had definite dimensions. This can be seen explicitly by inspecting the asymptotic solutions given in the appendix of [29]. Their exponential $\tau$ dependence $\left(\mathrm{e}^{(\Delta-4) \tau / 3}\right.$ for $\widehat{\mathfrak{a}}$ and $\mathrm{e}^{-\Delta \tau / 3}$ for $\left.\breve{\mathfrak{a}}\right)$ is what one would expect for a solution dual to an operator of dimension $\Delta$.) The KS radial variable $\tau$ will be introduced momentarily in (5.3).

The sigma-model metric is

$$
\begin{aligned}
G_{a b} \partial_{M} \phi^{a} \partial^{M} \phi^{b}= & \partial_{M} x \partial^{M} x+6 \partial_{M} p \partial^{M} p+\frac{1}{2} \partial_{M} y \partial^{M} y+\frac{1}{4} \partial_{M} \Phi \partial^{M} \Phi+\frac{P^{2}}{2} \mathrm{e}^{\Phi-2 x} \partial_{M} b \partial^{M} b \\
& +\frac{1}{4} \mathrm{e}^{-\Phi-2 x}\left[\mathrm{e}^{-2 y} \partial_{M}\left(h_{1}-h_{2}\right) \partial^{M}\left(h_{1}-h_{2}\right)+\mathrm{e}^{2 y} \partial_{M}\left(h_{1}+h_{2}\right) \partial^{M}\left(h_{1}+h_{2}\right)\right],
\end{aligned}
$$

and the superpotential reads

$$
W=-\frac{1}{2}\left(\mathrm{e}^{-2 p-2 x}+\mathrm{e}^{4 p} \cosh y\right)+\frac{1}{4} \mathrm{e}^{4 p-2 x}\left[Q+2 P\left(b h_{2}+h_{1}\right)\right] .
$$

Here, $Q$ and $P$ are constants related to the number of D3-branes and wrapped D5-branes, respectively.

It is useful to introduce the KS radial variable $\tau$ by

$$
\partial_{r}=\mathrm{e}^{4 p} \partial_{\tau}
$$


In terms of $\tau$, the KS background solution of (2.4) is given by

$$
\begin{gathered}
\Phi=\Phi_{0}, \\
\mathrm{e}^{y}=\tanh \left(\frac{\tau}{2}\right), \\
b=-\frac{\tau}{\sinh \tau}, \\
h_{1}=-\frac{Q}{2 P}+P \mathrm{e}^{\Phi_{0}} \operatorname{coth} \tau(\tau \operatorname{coth} \tau-1), \\
h_{2}=P \mathrm{e}^{\Phi_{0}} \frac{\tau \operatorname{coth} \tau-1}{\sinh \tau}, \\
\frac{2}{3} \mathrm{e}^{6 p+2 x}=\operatorname{coth} \tau-\frac{\tau}{\sinh ^{2} \tau}, \\
\mathrm{e}^{2 x / 3-4 p}=2 P^{2} \mathrm{e}^{\Phi_{0} 3^{-2 / 3} h(\tau) \sinh ^{4 / 3} \tau,}
\end{gathered}
$$

with

$$
h(\tau)=\int_{\tau}^{\infty} \mathrm{d} \vartheta \frac{\vartheta \operatorname{coth} \vartheta-1}{\sinh ^{2} \vartheta}[2 \sinh (2 \vartheta)-4 \vartheta]^{1 / 3}
$$

Moreover, the warp factor is given by

$$
\mathrm{e}^{-2 A} \sim \mathrm{e}^{4 p}\left(\mathrm{e}^{-2 x} \sinh \tau\right)^{2 / 3} h(\tau)
$$

with a proportionality factor that sets the momentum scale.

The KT background solution is somewhat simpler, because there $y=b=h_{2}=0$, but it has a singularity. For the KT background solutions of the other fields, we refer to [33].

\subsection{KS 7-Scalar System: Fluctuations}

Now, we consider fluctuations of the 7 scalars around the KS background. All scalars appear to be fully coupled in the bulk, but we can decouple a $4 \times 4$ set of fields from a $3 \times 3$ set to leading order in the UV, as will be discussed in a moment. The system of field equations we consider follows from (2.49) upon changing the radial coordinate to $\tau$. One finds

$$
\left[\left(\partial_{\tau}-M\right)\left(\partial_{\tau}-N\right)-k^{2} \mathrm{e}^{-2 A-8 p}\right] \mathfrak{a}=0
$$


where we have dropped the tensor indices, and the matrices $M$ and $N$ are defined by

$$
\begin{gathered}
M_{b}^{a}=-N_{b}^{a}-K_{b}^{a}-2 \mathrm{e}^{-2 x-6 p} \delta_{b}^{a}, \\
N_{b}^{a}=\mathrm{e}^{-4 p}\left(\partial_{b} W^{a}-\frac{W^{a} W_{b}}{W}\right), \\
K_{b}^{a}=2 \mathrm{e}^{-4 p} \mathcal{G}_{b c}^{a} W^{c} .
\end{gathered}
$$

We fix the momentum scale such that

$$
\mathrm{e}^{-2 A}=\mathrm{e}^{4 p}\left(\mathrm{e}^{-2 x} \sinh \tau\right)^{2 / 3} \frac{h(\tau)}{h(0)}
$$

The equations of motion (5.7) can be solved asymptotically (for large $\tau$ ), which is needed for the holographic renormalization procedure of chapter 3 . To do so one has to expand the matrices and the warp factor in powers of $\mathrm{e}^{-\tau}$. We spare the reader the technical details (which can be found in [29,45]) and just mention the following pattern in the asymptotic solutions. Let us consider the two groups of scalars consisting, on the one hand, of $x, p, h_{1}$ and $\Phi$, and on the other hand, of $y, b$ and $h_{2}$. (More precisely, we consider the gauge invariant scalars built on them according to (2.22).) In [45], these two sets of scalars were called the "glueball sector" and the "gluinoball sector", respectively. In the KT background, the scalars in the gluinoball sector are inert, that is, their background solutions are identically zero, and consequently any terms coupling the two sectors are absent. This eventually leads to the singularity in the IR, which is resolved in the KS background by taking into account the backreaction on the gluinoball sector. Nevertheless, the UV decoupling is also apparent in the asymptotic solutions. The solutions related to the operators of dimensions $\Delta=8,6$ and 4 only have the first four components excited at leading (and next-to-leading) order. These four components correspond exactly to the scalars of the glueball sector. The mixing only appears at order $\mathrm{e}^{-\tau}$ relative to the leading order. Similarly, the solutions related to operators of dimensions $\Delta=7$ and 3 only have the last three components excited at leading (and nextto-leading) order. These correspond to the scalars in the gluinoball sector.

\subsection{Holographic Renormalization}

We are now ready to apply the formalism of Section 3 to the case of the KS system. In the following discussion, we often restrict ourselves to the $\Delta \leq 4$ operators. This simplifies the calculations considerably, and it suffices to discuss all the general features of a system with several coupled scalars. We assume that the dominant and subdominant solutions are ordered in such a way that those dual to the $\Delta \leq 4$ operators carry the indices $i=4,5,6,7$.

In order to discuss the issue of scheme dependence, we allow for redefinitions of the dominant asymptotic solutions with the subdominant solutions. Again, we restrict to the $\Delta \leq 4$ operators and only modify the corresponding dominant solutions according to

$$
\widehat{\mathfrak{a}}_{i}^{\prime}=\widehat{\mathfrak{a}}_{i}+\lambda_{i}^{j} \check{\mathfrak{a}}_{j}
$$

with $i, j=4,5,6,7$. 
The matrix $Z$ from (3.4) can be calculated (using the solutions of the appendix in [29]) and is given by

$$
Z_{i j}=3^{1 / 3} P^{4} e^{2 \phi_{0}}\left(\begin{array}{ccccccc}
-\frac{80}{3} & 0 & \frac{5}{4} \beta & 0 & -\frac{2531}{192} \beta^{2} & -\frac{19}{4} \beta & \frac{419}{80} \beta \\
0 & -\frac{2}{9} & 0 & 0 & \frac{8}{3} & \frac{3337}{11520} \beta^{2} & \frac{6913}{3200} \beta^{2} \\
0 & 0 & \frac{20}{9} & \frac{737}{120} \beta & -\frac{4439}{600} \beta & -\frac{76}{9} & -\frac{7}{15} \\
0 & 0 & 0 & -\frac{4}{9} & \frac{10}{9} & 0 & 0 \\
0 & 0 & 0 & 0 & \frac{4}{9} & 0 & 0 \\
0 & 0 & 0 & 0 & 0 & -\frac{2}{9} & \frac{5}{9} \\
0 & 0 & 0 & 0 & 0 & 0 & \frac{4}{9}
\end{array}\right) \text {, }
$$

where we have introduced the abbreviation

$$
\beta=\frac{3^{1 / 3}}{h(0)} k^{2}
$$

Note that $Z$ does not depend on the $\lambda_{i}^{j}$, that is, it is scheme independent as it should be according to (3.25).

As discussed in Section 3.3, one can also redefine the dominant solutions by other dominant ones. In particular, to a dominant solution of dimension $\Delta$, one could add other dominant solutions of dimensions smaller than or equal to $\Delta$. This would amount to an upper triangular matrix $\Lambda$, compare for example, (3.24). It is easy to check that using $\mu_{i}^{j}=\delta_{i}^{j}$ and

$$
\Lambda_{i}^{j}=\left(3^{1 / 3} P^{4} \mathrm{e}^{2 \phi_{0}}\right)^{-1}\left(\begin{array}{ccccccc}
-\frac{3}{80} & 0 & \frac{27}{1280} \beta & \frac{59697}{204800} \beta^{2} & -\frac{3051207}{2048000} \beta^{2} & 0 & \frac{297}{640} \beta \\
0 & -\frac{9}{2} & 0 & 0 & 27 & -\frac{30033}{5120} \beta^{2} & \frac{2990637}{102400} \beta^{2} \\
0 & 0 & \frac{9}{20} & \frac{19899}{3200} \beta & -\frac{257769}{32000} \beta & -\frac{171}{10} & \frac{8739}{400} \\
0 & 0 & 0 & -\frac{9}{4} & \frac{45}{8} & 0 & 0 \\
0 & 0 & 0 & 0 & \frac{9}{4} & 0 & 0 \\
0 & 0 & 0 & 0 & 0 & -\frac{9}{2} & \frac{45}{8} \\
0 & 0 & 0 & 0 & 0 & 0 & \frac{9}{4}
\end{array}\right)
$$


in (3.25), which also rescales all operators, would lead to a matrix

$$
Z_{i j}^{\prime}=\delta_{i j}
$$

Let us mention in passing that the appearance of the $\beta$-factors in (5.13) leads to a "natural" form of $\Lambda_{i}^{j}$, according to the discussion in Section 3.3. It ensures that the structure of the dominant solutions stays intact, that is, also after the redefinition the same combinations of $\beta$ and $\mathrm{e}^{\tau}$ appear as before. (Of course, this only becomes apparent by looking at the explicit form of the asymptotic solutions given in the appendix of [29].)

Let us turn to the matrix $\widetilde{Z}$ from (3.4). As stated in Section 3.3, one can always choose a basis such that $\tilde{Z}_{i j}=0$. This statement holds also after the operator redefinition given by (5.13). Allowing for scheme dependence in the rotated basis, one would find from (3.25)

$$
\tilde{Z}_{i j}^{\prime}=\lambda_{j i}-\lambda_{i j}
$$

Calculating the two-point functions of the dual operators using (3.8) is, of course, more involved and goes beyond the scope of this article. It would be interesting to make the scaling with $N_{\text {eff }} \sim \ln (k / \Lambda)$ explicit, where $\Lambda$ is the confinement scale, but certainly one would have to rely on numerical methods. We note that in $[39,40]$ an approximation method was devised to determine the leading order term of the two-point functions in an expansion for large momenta. This method was also applied in $[33,41]$. It would be interesting to see whether and how the renormalization procedure presented here would modify the results of $[39,40]$. Another remark is that, in any case, the renormalization procedure devised here justifies $a$ posteriori the pragmatic approach taken in [45] for the calculation of mass spectra in the KS system. Probably, it also applies to the calculations in $[46,47]$ if it is possible to bring the system considered there into the form of a fake SUGRA system.

It is also interesting to consider the counterterm matrix $U_{a b}$. It is rather complicated, and we only give the leading terms in an expansion in $\epsilon=\mathrm{e}^{-2 \tau / 3}$. For $\lambda_{i j}=0$, it is given by

$$
U_{a b}=2^{1 / 3}\left(\frac{\mathrm{e}^{-\phi_{0}}}{P^{2}(4 \tau-1)}\right)^{2 / 3}\left(\begin{array}{ll}
U_{4 \times 4} & U_{4 \times 3} \\
U_{3 \times 4} & U_{3 \times 3}
\end{array}\right)
$$

with the submatrices

$$
U_{4 \times 4}=\left(\begin{array}{cccc}
-\frac{32}{15} & -\frac{32}{5} & -\frac{9}{640}(\varepsilon) \beta^{2} \epsilon^{2} & -\frac{9 \beta \epsilon}{20} \\
-\frac{32}{5} & -\frac{96}{5} & 3 \beta \epsilon & -\frac{117}{20} \beta \epsilon \\
-\frac{9}{640}(\varepsilon) \beta^{2} \epsilon^{2} & 3 \beta \epsilon & -\beta \epsilon & \frac{3}{2} \beta \epsilon \\
-\frac{9}{20} \beta \epsilon & -\frac{117}{20} \beta \epsilon & \frac{3}{2} \beta \epsilon & -\frac{3(4 \tau+17) \beta \epsilon}{16}
\end{array}\right),
$$




$$
\begin{gathered}
U_{3 \times 4}=U_{4 \times 3}^{T}=\frac{\epsilon^{3 / 2}}{4 \tau+5}\left(\begin{array}{cccc}
\frac{4}{5}(28 \tau-31) & \frac{128}{5}(2 \tau+1) & \frac{16}{3} & \mathcal{O}(\epsilon) \\
\frac{16}{15}(2 \tau+19) & \frac{256}{15}(\tau+2) & -\frac{16}{9} & \mathcal{O}(\epsilon) \\
-\frac{16}{15}(2 \tau+19) & -\frac{256}{15}(\tau+2) & \frac{16}{9} & \mathcal{O}(\epsilon)
\end{array}\right), \\
U_{3 \times 3}=\frac{1}{4 \tau+5}\left(\begin{array}{ccc}
-\frac{2}{3}(4 \tau+17) & \frac{8}{3} & -\frac{8}{3} \\
\frac{8}{3} & -\frac{8}{9} & \frac{8}{9} \\
-\frac{8}{3} & \frac{8}{9} & -\frac{8}{9}
\end{array}\right)
\end{gathered}
$$

where $\varepsilon$ denotes $32 \tau^{2}+148 \tau-873$.

The entries of $U_{3 \times 4}$ and $U_{4 \times 3}$ lead to mixings between the fields in the glueball and gluinoball sectors. When considering nonvanishing $\lambda_{i j}$, one notices that they are all schemedependent.

In general, the scheme-dependent terms should only lead to finite contributions to the action. We have verified this explicitly for the $\Delta \leq 4$ operators. Using the counterterm matrix with $\lambda_{i j} \neq 0$ and considering nonvanishing sources only for the operators with $\Delta \leq 4$, we find

$$
\mathrm{e}^{4 A} \mathfrak{a} \cdot U \cdot \mathfrak{a}=\sum_{i, j=4}^{7} \mathfrak{s}^{i}\left(V_{i j}^{(1)}\left(\lambda_{k l}\right)+\epsilon^{-1} V_{i j}^{(2)}+V_{i j}^{(3)}\right) \mathfrak{s}^{j}
$$

with

$$
\begin{aligned}
& \left.V^{(1)}\right|_{4-7}=\frac{1}{9} 3^{1 / 3} P^{4} \mathrm{e}^{2 \phi_{0}} \\
& \times\left(\begin{array}{cccc}
10 \lambda_{45}-4 \lambda_{44} & 2 \lambda_{45}-2 \lambda_{54}+5 \lambda_{55} & \frac{5}{2} \lambda_{47}+5 \lambda_{65}-2 \lambda_{64}-\lambda_{46} & 2 \lambda_{47}-2 \lambda_{74}+5 \lambda_{75} \\
2 \lambda_{45}-2 \lambda_{54}+5 \lambda_{55} & 4 \lambda_{55} & \frac{5}{2} \lambda_{57}-\lambda_{56}+2 \lambda_{65} & 2 \lambda_{57}+2 \lambda_{75} \\
\frac{5}{2} \lambda_{47}+5 \lambda_{65}-2 \lambda_{64}-\lambda_{46} & \frac{5}{2} \lambda_{57}-\lambda_{56}+2 \lambda_{65} & 5 \lambda_{67}-2 \lambda_{66} & \frac{5}{2} \lambda_{77}+2 \lambda_{67}-\lambda_{76} \\
2 \lambda_{47}-2 \lambda_{74}+5 \lambda_{75} & 2 \lambda_{57}+2 \lambda_{75} & \frac{5}{2} \lambda_{77}+2 \lambda_{67}-\lambda_{76} & 4 \lambda_{77}
\end{array}\right) .
\end{aligned}
$$

These finite terms are analogous to the finite quartic counterterms in the GPPZ flow and the finite quadratic counterterm in the CB flow, compare for example, (4.20) and (4.42), respectively, after expanding them to quadratic order in the fluctuations. 
In addition to these finite terms there are also divergent contributions which are either linearly diverging in $\epsilon=\mathrm{e}^{-2 \tau / 3}$ or logarithmically. These are given by

$$
\begin{gathered}
\left.V^{(2)}\right|_{4-7}=\frac{1}{9} 3^{1 / 3} P^{4} \mathrm{e}^{2 \phi_{0}} \times\left(\begin{array}{cccc}
-\frac{3}{2}\left(\tau^{2}-3 \tau+5\right) \beta & -\frac{3}{8}(4 \tau-7) \beta & 0 & 0 \\
-\frac{3}{8}(4 \tau-7) \beta & -3 \frac{2 \tau+1}{4 \tau+1} \beta & 0 & 0 \\
0 & 0 & -\frac{1}{4}\left(16 \tau^{2}+28 \tau+19\right) & -4(2 \tau+1) \\
0 & 0 & -4(2 \tau+1) & -16
\end{array}\right) \\
\left.V^{(3)}\right|_{4-7}=\frac{1}{9} 3^{1 / 3} P^{4} \mathrm{e}^{2 \phi_{0}}\left(\begin{array}{cc}
V^{(3, \Delta=4)} & 0_{2 \times 2} \\
0_{2 \times 2} & V^{(3, \Delta=3)}
\end{array}\right),
\end{gathered}
$$

with

$$
\begin{aligned}
& V^{(3, \Delta=4)}=\beta^{2}\left(\begin{array}{cc}
\frac{9}{16} \tau^{4}+\frac{75}{16} \tau^{3}+\frac{189}{32} \tau^{2}-\frac{5355}{128} \tau+\frac{38979}{256} & \frac{3}{256} \tau \\
\frac{3}{256} \tau & \frac{9}{64} \frac{32 \tau^{3}+424 \tau^{2}+916 \tau+371}{4 \tau+1}
\end{array}\right), \\
& V^{(3, \Delta=3)}=\beta\left(\begin{array}{cc}
\frac{3}{2} \tau^{4}+\frac{45}{2} \tau^{3}+\frac{477}{4} \tau^{2}+\frac{1959}{8} \tau-\frac{1239}{32} & 4 \tau^{3}+51 \tau^{2}+\frac{975}{4} \tau-\frac{831}{16} \\
4 \tau^{3}+51 \tau^{2}+\frac{975}{4} \tau-\frac{831}{16} & 12 \tau^{2}+156 \tau+213
\end{array}\right),
\end{aligned}
$$

where $\tau$ denotes $\left(256 \tau^{4}+2368 \tau^{3}+4368 \tau^{2}-5664 \tau-1821\right) /(4 \tau+1)$. Note that the linear divergences are momentum independent for the $\Delta=3$ operators and proportional to $k^{2}$ for the $\Delta=4$ operators. Furthermore, the logarithmically divergent terms are proportional to $k^{2}$ for the $\Delta=3$ operators and proportional to $k^{4}$ for the $\Delta=4$ operators. All this is very reminiscent of the aAdS case, compare for example, Sections 5.2 and 5.3 in [12]. There is, however, a difference in the fact that the logarithms appear in a much more complicated way, and they are even present in the linearly divergent terms. Although some of this may be an artifact of the choice of radial variable, this is consistent with the fact that the KS theory has no UV conformal fixed point.

As mentioned above, all the entries of $U_{3 \times 4}$ and $U_{4 \times 3}$ are scheme-dependent and thus only contribute finite terms to the renormalized action. This implies that one could have determined all the divergent terms for the glueball-sector and the gluinoball-sector separately. In other words, one can renormalize the KT theory without embedding it into the KS theory. This is plausible, as the KT background is a good approximation to the KS background in the asymptotic region, and the field theory divergences are UV divergences. 
This standpoint was also taken in [30,41], where the renormalization was performed just for the KT background.

\subsection{Gluino Condensate}

Finally, we would like to comment on the issue of VEVs. As we saw in the aAdS cases of Section 4 , the response function of the background fluctuation $W_{\phi} / W$ encodes the VEV in those cases (up to an overall factor). We would like to see how this carries over to the case of KS, where we expect a nonzero VEV for a gluinoball operator of $\Delta=3$. In order to derive the VEV from first principles, one would need the exact form of the counterterms linear in the fluctuations, which we have not determined yet. Thus, we can only take the cases of GPPZ and CB as encouraging examples and calculate, in analogy, the response coefficients of $W^{a} / W$. It is straightforward to calculate

$$
\frac{W^{a}}{W}=\frac{4}{4 \tau+1}\left(\begin{array}{c}
-1 \\
\frac{1}{3} \\
-2\left(\tau-\frac{1}{4}\right) \\
0 \\
0_{3}
\end{array}\right)+\frac{4 \mathrm{e}^{-\tau}}{4 \tau+1}\left(\begin{array}{c}
\mathbf{0}_{4} \\
-4 \tau+1 \\
(-4 \tau+1)(\tau-1) \\
(\tau-2)(4 \tau-1)
\end{array}\right)+\mathcal{O}\left(\mathrm{e}^{-2 \tau}\right) \text {. }
$$

Comparing this with the asymptotic solutions, we obtain

$$
\frac{W^{a}}{W}=-2 \widehat{\mathfrak{a}}_{5}-4 \check{\mathfrak{a}}_{7}+2 \check{\mathfrak{a}}_{6},
$$

where $\widehat{\mathfrak{a}}_{5}$ is the dominant solution corresponding to one of the $\Delta=4$ operators and $\check{\mathfrak{a}}_{6}$ and $\check{\mathfrak{a}}_{7}$ are the subdominant solutions of the $\Delta=3$ operators. This result suggests the interpretation that a combination of the two $\Delta=3$ operators has a VEV, which is in agreement with the field theory expectation of a condensate of the gluino bilinear [34, 70]. However, this statement is again scheme-dependent. The redefinition (5.10) leads to

$$
\mathfrak{a}=\mathfrak{s}^{i} \widehat{\mathfrak{a}}_{i}+\mathfrak{r}^{i} \check{\mathfrak{a}}_{i}=\mathfrak{s}^{i} \widehat{\mathfrak{a}}_{i}^{\prime}+\left(\mathfrak{r}^{i}-\mathfrak{s}^{j} \lambda_{j}^{i}\right) \check{\mathfrak{a}}_{i}
$$

and applying this to $W^{a} / W$ results in

$$
\frac{W^{a}}{W}=-2 \widehat{\mathfrak{a}}_{5}+\left(-4+2 \lambda_{57}\right) \check{\mathfrak{a}}_{7}+\left(2+2 \lambda_{56}\right) \check{\mathfrak{a}}_{6}+2 \lambda_{55} \check{\mathfrak{a}}_{5}+2 \lambda_{54} \check{\mathfrak{a}}_{4},
$$

$\check{\mathfrak{a}}_{4}$ and $\check{\mathfrak{a}}_{5}$ being the subdominant solutions of the $\Delta=4$ operators. Let us apply the "naturalness" criterion on the form of the $\lambda_{i j}$ described in Section 3.3. It would give $\lambda_{55}, \lambda_{54} \sim$ $\beta^{2}$, but $\beta=0$ in (5.25), so that the coefficients of $\check{\mathfrak{a}}_{4}$ and $\check{\mathfrak{a}}_{5}$ vanish. The coefficients of the $\check{\mathfrak{a}}_{6}$ and $\check{\mathfrak{a}}_{7}$ belonging to the $\Delta=3$ operators are more subtle, because the $\mathrm{e}^{-\tau}$ term in 
$\widehat{\mathfrak{a}}_{5}$, which is the leading term in $\check{\mathfrak{a}}_{6}$ and $\check{\mathfrak{a}}_{7}$, is independent of $\beta$. On physical grounds we expect that there should be a natural scheme in which the VEVs for the $\Delta=3$ operators are not both vanishing simultaneously, compare for example, [34, 70]. It would be very interesting to understand how to determine such a preferred scheme, which might amount to extending the "naturalness" criterion of Section 3.3 or to finding an equivalent of the supersymmetric scheme in the GPPZ flow. Obviously, it would also be interesting to obtain the VEVs independently, using the linear terms of the action, but for this one would need the linear counterterms.

\section{Conclusions and Outlook}

The recent efforts to perform HR systematically in nonaAdS backgrounds, which we have reviewed, offer a promising picture for cascading gauge theories, like KS. It appears that the pragmatic approach taken in various calculations of glueball mass spectra is justified, that is, it has been established that two-point functions of renormalized operators exist, which exhibit precisely the calculated mass spectra. This follows, as they are indeed of the general form (3.9) assumed in the calculation of the spectra. We have presented the case of scalar fields, but a generalization to other independent gauge-invariant fluctuations, for example, the transverse traceless metric fluctuations, seems straightforward. The new order-by-order approach, devised in [29], nicely reproduces the results of HR in aAdS settings. In particular, the scheme dependence is incorporated in an interesting fashion.

There is, obviously, much room for further development. First, one needs to understand the role of the gauge-dependent terms that remain in the full on-shell action. The action (3.1) appears, of course, in an expansion of (2.1), but gauge-dependent secondorder terms involving the field $h$, especially, have been dropped. In aAdS settings, finite terms of this kind are related to a conformal anomaly. A related question is whether the second-order counterterms can be obtained from an expansion of some covariant expression of the full fields, from which one may deduce Ward identities between various correlation functions. Presumably, this would also shed some light on the question how to calculate the VEV. Indeed, adding the superpotential $W$ as a boundary term removes terms linear in the fluctuations from the action. Therefore, a VEV must stem from the counterterms, which is precisely how it works in aAdS settings. There, the counterterm $U(\phi)[7,11]$ may differ from the superpotential $W(\phi)$, although it satisfies the same equation (2.2). Expanding the difference $U(\phi)-W(\phi)$ to first order in the fluctuations yields precisely the VEV.

Second, it would be very interesting to find a better definition of the naturalness criterion for the choice of the asymptotically dominant basis solutions. This question is essential to limit this choice to the freedom of choosing a scheme that one has in QFT. We have seen in the examples in Section 4 that the scheme dependence yields redefinitions of the dominant basis with certain powers of $k^{2}$. Such a naturalness criterion may offer an alternative way to unambiguously determine VEVs.

Third, one should investigate how the method extends to higher-orders in the fluctuations. The quadratic terms in the bulk equations of motion have been given in [33], including the metric fluctuations. From these, one may determine three-point functions and scattering amplitudes.

Last, one should find a formulation of HR for systems, in which the assumptions on the form of the asymptotic basis solutions do not hold. For the KS case considered here they are satisfied, but other interesting cases in which people have used a pragmatic approach for 
mass spectra, for example, the Maldacena-Nunez system $[45,48]$ or the walking backgrounds $[50,54]$, have a very different behaviour. It would also be interesting to see whether and, if yes, how the systems considered in [22] could be described in the present approach.

\section{Acknowledgments}

It is a pleasure to thank M. Berg and N. Borodatchenkova for their collaboration on the various topics covered in this paper. Michael Haack is supported in part by the Excellence Cluster "The Origin and the Structure of the Universe" in Munich and by the German Research Foundation (DFG) within the Emmy-Noether-Program (Grant no.: HA 3448/3-1). The work of Wolfgang Mück was partially supported by the INFN research initiative TV12, as well as by the Italian Ministry of Education and Research (MIUR), PRIN project 2005-023102. Michael Haack would like to thank the KITP in Santa Barbara for hospitality during his work on this review.

\section{References}

[1] J. M. Maldacena, "The large n limit of superconformal field theories and supergravity," Advances in Theoretical and Mathematical Physics, vol. 2, pp. 231-252, 1998.

[2] S. S. Gubser, I. R. Klebanov, and A. M. Polyakov, "Gauge theory correlators from non-critical string theory," Physics Letters B, vol. 428, no. 1-2, pp. 105-114, 1998.

[3] E. Witten, "Anti-de Sitter space and holography," Advances in Theoretical and Mathematical Physics, vol. 2, pp. 253-291, 1998.

[4] M. Abramowitz and I. A. Stegun, Eds., Handbook of Mathematical Functions, Dover Publications, New York, NY, USA, 1965.

[5] M. Henningson and K. Skenderis, "The holographic Weyl anomaly," Journal of High Energy Physics, vol. 2, no. 7, article 023, 1998.

[6] V. Balasubramanian and P. Kraus, "A stress tensor for anti-de Sitter gravity," Communications in Mathematical Physics, vol. 208, no. 2, pp. 413-428, 1999.

[7] J. de Boer, E. Verlinde, and H. Verlinde, “On the holographic renormalization group," Journal of High Energy Physics, vol. 4, no. 8, pp. 1-15, 2000.

[8] S. de Haro, K. Skenderis, and S. N. Solodukhin, "Holographic reconstruction of spacetime and renormalization in the AdS/CFT correspondence," Communications in Mathematical Physics, vol. 217, no. 3, pp. 595-622, 2001.

[9] J. Kalkkinen, D. Martelli, and W. Mück, "Holographic renormalisation and anomalies," Journal of High Energy Physics, vol. 5, no. 4, 2001.

[10] M. Bianchi, D. Z. Freedman, and K. Skenderis, "Holographic renormalization," Nuclear Physics B, vol. 631, no. 1-2, pp. 159-194, 2002.

[11] D. Martelli and W. Mück, "Holographic renormalization and Ward identities with the HamiltonJacobi method," Nuclear Physics B, vol. 654, no. 1-2, pp. 248-276, 2003.

[12] K. Skenderis, "Lecture notes on holographic renormalization," Classical and Quantum Gravity, vol. 19, no. 22, pp. 5849-5876, 2002.

[13] I. Papadimitriou and K. Skenderis, “AdS/CFT correspondence and geometry,” http://arxiv.org/abs/ hep-th/0404176.

[14] I. Papadimitriou and K. Skenderis, "Correlation functions in holographic RG flows," Journal of High Energy Physics, vol. 8, no. 10, pp. 2531-2567, 2004.

[15] K. Skenderis and B. C. V. Rees, "Real-time gauge/gravity duality: prescription, renormalization and examples," Journal of High Energy Physics, vol. 2009, no. 5, article 085, 2009.

[16] M. Taylor, "Non-relativistic holography," http://arxiv.org/abs/0812.0530.

[17] M. Guica, K. Skenderis, M. Taylor, and B. van Rees, "Holography for Schrodinger backgrounds," http://arxiv.org/abs/1008.1991.

[18] S. F. Ross and O. Saremi, "Holographic stress tensor for non-relativistic theories," Journal of High Energy Physics, vol. 2009, no. 9, article 009, 2009. 
[19] K. Balasubramanian and J. McGreevy, "An analytic Lifshitz black hole," Physical Review D, vol. 80, no. 10, Article ID 104039, 2009.

[20] P. Horava and C. M. Melby-Thompson, "Anisotropic Conformal Infinity," http://arxiv.org/abs/ 0909.3841.

[21] A. Karch, A. O’Bannon, and K. Skenderis, "Holographic renormalization of probe D-branes in AdS/CFT," Journal of High Energy Physics, vol. 2006, no. 4, pp. 349-371, 2006.

[22] I. Kanitscheider, K. Skenderis, and M. Taylor, "Precision holography for non-conformal branes," Journal of High Energy Physics, vol. 2008, no. 9, article 094, 2008.

[23] D. Z. Freedman, C. Núñez, M. Schnabl, and K. Skenderis, "Fake supergravity and domain wall stability," Physical Review D, vol. 69, no. 10, Article ID 104027, 2004.

[24] A. Celi, A. Ceresole, G. Dall'Agata, A. Van Proeyen, and M. Zagermann, "On the fakeness of fake supergravity," Physical Review D, vol. 71, no. 4, Article ID 045009, 16 pages, 2005.

[25] M. Zagermann, "N=4 "fake" supergravity," Physical Review D, vol. 71, no. 12, Article ID 125007, 2005.

[26] O. Aharony, "The non-AdS/non-CFT correspondence, or three different paths to QCD," http:/ /arxiv .org/abs/hep-th/0212193.

[27] A. Zaffaroni, "RTN lectures on the non AdS / non CFT correspondence," PoS RTN2005(2005) 005.

[28] J. Erdmenger, N. Evans, I. Kirsch, and E. J. Threlfall, "Mesons in gauge/gravity duals," The European Physical Journal A, vol. 35, no. 1, pp. 81-133, 2008.

[29] N. Borodatchenkova, M. Haack, and W. Mück, "Towards holographic renormalization of fake supergravity," Nuclear Physics B, vol. 815, no. 1-2, pp. 215-239, 2009.

[30] O. Aharony, A. Buchel, and A. Yarom, "Holographic renormalization of cascading gauge theories," Physical Review D, vol. 72, no. 6, Article ID 066003, 30 pages, 2005.

[31] M. Bianchi, M. Prisco, and W. Mück, "New results on holographic three-point functions," Journal of High Energy Physics, vol. 7, no. 11, pp. 1163-1186, 2003.

[32] W. Mück, "Progress on holographic three-point functions," Fortschritte der Physik, vol. 53, no. 7-8, pp. 948-954, 2005.

[33] M. Berg, M. Haack, and W. Mück, "Bulk dynamics in confining gauge theories," Nuclear Physics B, vol. 736, no. 1-2, pp. 82-132, 2006.

[34] I. R. Klebanov and M. J. Strassler, "Supergravity and a confining gauge theory: duality cascades and xSB-resolution of naked singularities," Journal of High Energy Physics, vol. 4, no. 8, pp. 21-35, 2000.

[35] I. R. Klebanov and A. A. Tseytlin, "Gravity duals of supersymmetric SU(N) $\times \mathrm{SU}(\mathrm{N}+\mathrm{M})$ gauge theories," Nuclear Physics B, vol. 578, no. 1-2, pp. 123-138, 2000.

[36] G. Papadopoulos and A. A. Tseytlin, "Complex geometry of conifolds and a 5-brane wrapped on a 2-sphere," Classical and Quantum Gravity, vol. 18, no. 7, pp. 1333-1353, 2001.

[37] D. Cassani and A. F. Faedo, "A supersymmetric consistent truncation for conifold solutions," http://arxiv.org/abs/1008.0883.

[38] I. Bena, G. Giecold, M. Grana, N. Halmagyi, and F. Orsi, "Supersymmetric consistent truncations of IIB on T(1,1)," http:/ / arxiv.org/abs/1008.0983.

[39] M. Krasnitz, "A two point function in a cascading $\mathrm{N}=1$ gauge theory from supergravity," http://arxiv.org/abs/hep-th/0011179.

[40] M. Krasnitz, "Correlation functions in a cascading $N=1$ gauge theory," Journal of High Energy Physics, vol. 6, no. 12, pp. 987-1015, 2002.

[41] O. Aharony, A. Buchel, and A. Yarom, "Short distance properties of cascading gauge theories," Journal of High Energy Physics, vol. 2006, no. 11, article no. 069, 2006.

[42] M. Mia, K. Dasgupta, C. Gale, and S. Jeon, "Five easy pieces: the dynamics of quarks in strongly coupled plasmas," Nuclear Physics B, vol. 839, no. 1-2, pp. 187-293, 2010.

[43] E. Cáceres and R. Hernández, "Glueball masses for the deformed conifold theory," Physics Letters B, vol. 504, no. 1-2, pp. 64-70, 2001.

[44] E. Cáceres and X. Amador, "Spin two glueball mass and glueball regge trajectory from supergravity," Journal of High Energy Physics, vol. 8, no. 11, pp. 627-638, 2004

[45] M. Berg, M. Haack, and W. Mück, "Glueballs vs. gluinoballs: fluctuation spectra in non-AdS/nonCFT," Nuclear Physics B, vol. 789, no. 1-2, pp. 1-44, 2008.

[46] M. K. Benna, A. Dymarsky, I. R. Klebanov, and A. Solovyov, "On normal modes of a warped throat," Journal of High Energy Physics, vol. 2008, no. 6, article 070, 2008.

[47] A. Dymarsky, D. Melnikov, and A. Solovyov, "I-odd sector of the Klebanov-Strassler theory," Journal of High Energy Physics, vol. 2009, no. 5, article 105, 2009.

[48] J. Maldacena and C. Nuñez, "Towards the large N limit of pure N = 1 super Yang-Mills theory," Physical Review Letters, vol. 86, no. 4, pp. 588-591, 2001. 
[49] V. A. Novikov, M. A. Shifman, A. I. Vainshtein, and V. I. Zakharov, "Exact Gell-Mann-Low function of supersymmetric Yang-Mills theories from instanton calculus," Nuclear Physics B, vol. 229, no. 2, pp. 381-393, 1983.

[50] C. Núñez, I. Papadimitriou, and M. Piai, "Walking dynamics from string duals," International Journal of Modern Physics A, vol. 25, no. 14, pp. 2837-2865, 2010.

[51] O. C. Gürdoğan, "Walking solutions in the string background dual to N = 1 SQCD-like theories," Annals of Physics, vol. 325, no. 3, pp. 535-547, 2010.

[52] M. Piai, "Lectures on walking technicolor, holography and gauge/gravity dualities," http://arxiv .org/abs/1004.0176.

[53] T. Appelquist, D. Karabali, and L. C. R. Wijewardhana, "Chiral hierarchies and flavor-changing neutral currents in hypercolor," Physical Review Letters, vol. 57, no. 8, pp. 957-960, 1986.

[54] D. Elander, C. Núñez, and M. Piai, "A light scalar from walking solutions in gauge-string duality," Physics Letters B, vol. 686, no. 1, pp. 64-67, 2010.

[55] D. Elander, "Glueball spectra of SQCD-like theories," Journal of High Energy Physics, vol. 2010, no. 3, article 114, 2010.

[56] L. Girardello, M. Petrini, M. Porrati, and A. Zaffaroni, "Novel local CFT and exact results on perturbations of $\mathrm{N}=4$ super Yang Mills from AdS dynamics," Journal of High Energy Physics, vol. 2, no. 12 , article 022,1998 .

[57] D. Z. Freedman, S. S. Gubser, K. Pilch, and N. P. Warner, “Continuous distributions of D3-branes and gauged supergravity," Journal of High Energy Physics, vol. 4, no. 7, pp. 1-17, 2000.

[58] A. Brandhuber and K. Sfetsos, "Wilson loops from multicentre and rotating branes, mass gaps and phase structure in gauge theories," Advances in Theoretical and Mathematical Physics, vol. 3, pp. 851-887, 1999.

[59] O. DeWolfe and D. Z. Freedman, "Notes on fluctuations and correlation functions in holographic renormalization group flows," http://arxiv.org/abs/hep-th/0002226.

[60] G. Arutyunov, S. Frolov, and S. Theisen, "Gravity-scalar fluctuations in holographic RG flow geometries," Physics Letters B, vol. 484, no. 3-4, pp. 295-305, 2000.

[61] W. Mück, "Correlation functions in holographic renormalization group flows," Nuclear Physics B, vol. 620, no. 3, pp. 477-500, 2002.

[62] R. M. Wald, General Relativity, University of Chicago Press, Chicago, Ill, USA, 1984.

[63] A. Z. Petrov, Einstein Spaces, Pergamon Press, Oxford, UK, 1969.

[64] C. W. Misner, K. S. Thorne, and J. A. Wheeler, Gravitation, Freeman, San Francisco, Calif, USA, 1973.

[65] M. Bianchi, O. DeWolfe, D. Z. Freedman, and K. Pilch, "Anatomy of two holographic renormalization group flows," Journal of High Energy Physics, vol. 5, no. 1, article 021, 2001.

[66] W. Mück and M. Prisco, "Glueball scattering amplitudes from holography," Journal of High Energy Physics, vol. 8, no. 4, pp. 835-869, 2004.

[67] M. Blanchi, "How to go with an RG Flow," Journal of High Energy Physics, vol. 5, no. 8, article 041, 2001.

[68] S. S. Gubser, I. R. Klebanov, and C. P. Herzog, "Symmetry breaking and axionic strings in the warped deformed conifold," Journal of High Energy Physics, vol. 8, no. 9, pp. 795-820, 2004.

[69] S. S. Gubser, C. P. Herzog, and I. R. Klebanov, "Variations on the warped deformed conifold," Comptes Rendus Physique, vol. 5, no. 9-10, pp. 1031-1038, 2004.

[70] A. Loewy and J. Sonnenschein, "On the holographic duals of N = 1 gauge dynamics," Journal of High Energy Physics, vol. 5, no. 8, article 007, 2001. 

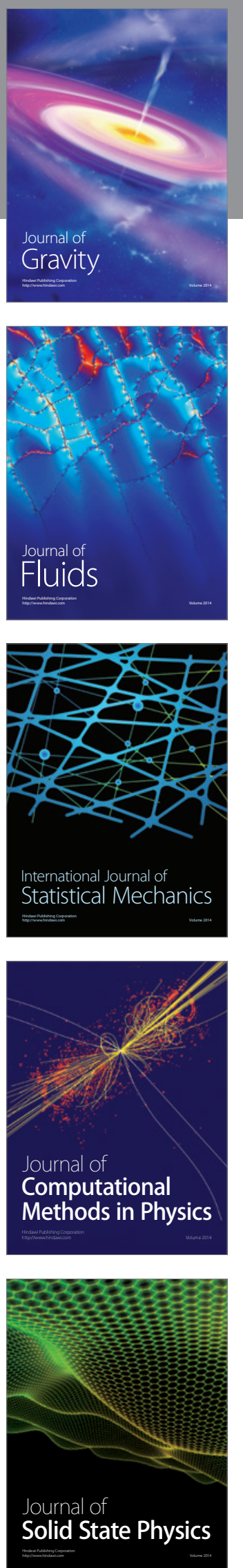

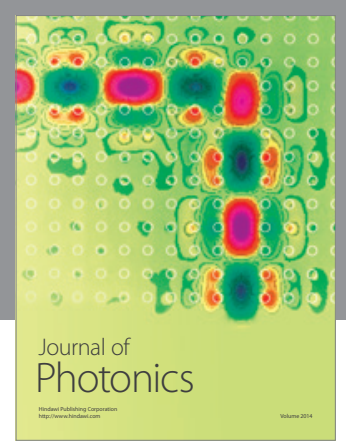

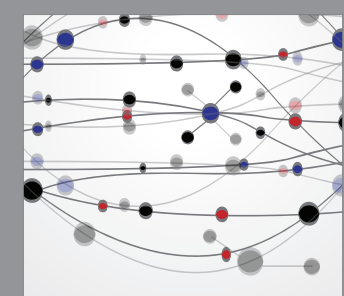

The Scientific World Journal
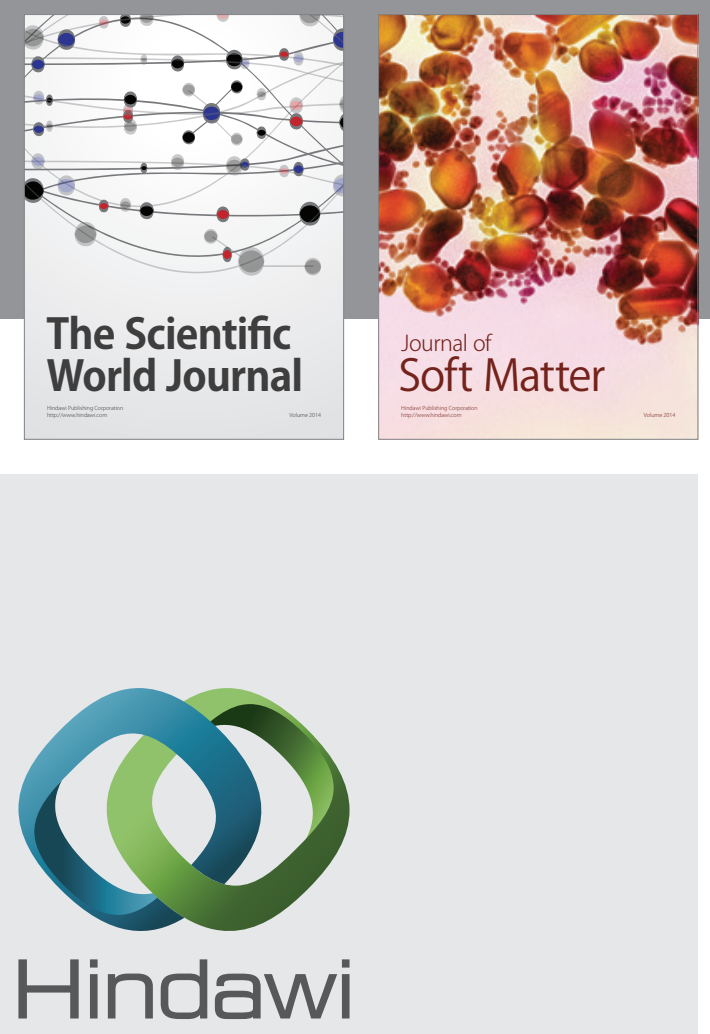

Submit your manuscripts at

http://www.hindawi.com
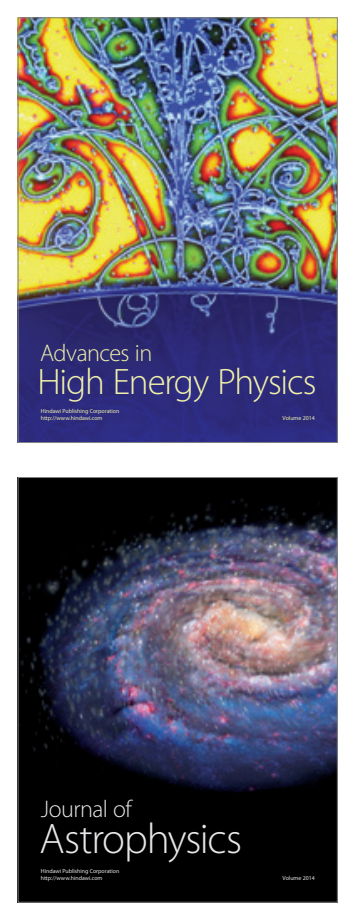
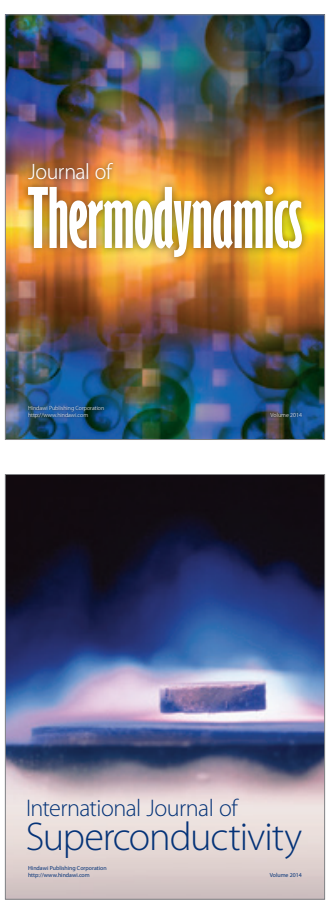
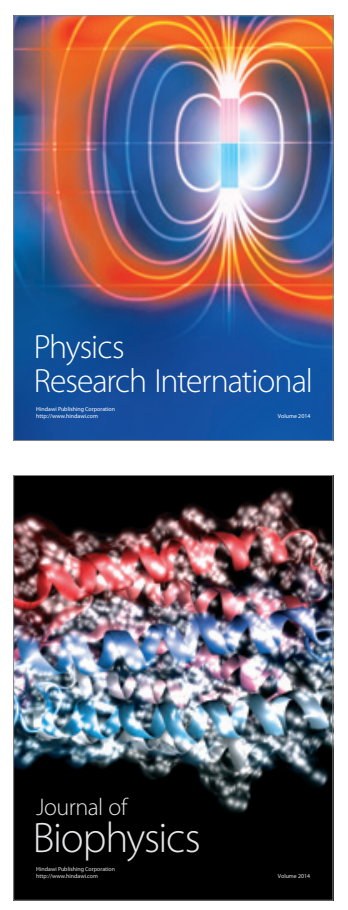
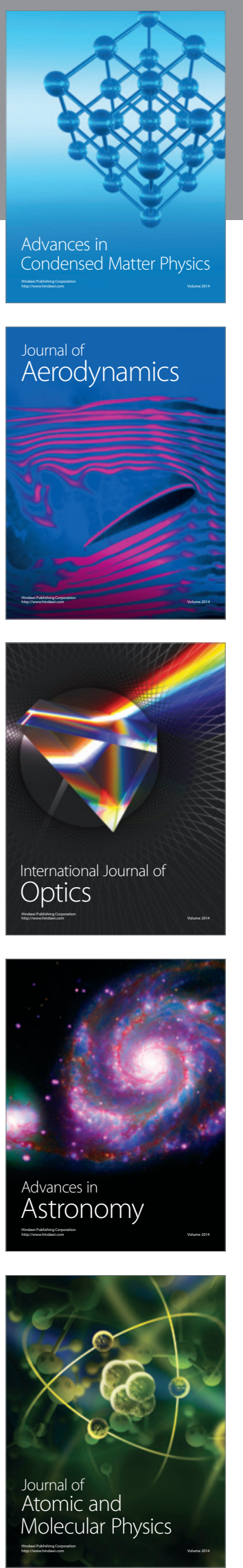